\title{
Simulation and Experimental Research on the Cooling Medium of Diamond Single Particles and Beads Cutting Reinforced Concrete
}

\section{Lan Zhang}

Harbin Engineering University

\section{Yongyin Pang}

Harbin Engineering University

Ming Liu ( $\sim$ lium@hrbeu.edu.cn )

Harbin Engineering University

\section{Liquan Wang}

Harbin Engineering University

\section{Xianbin Sha}

Harbin Engineering University

\section{Yue Xiao}

Harbin Engineering University

\section{Original Article}

Keywords: Diamond Wire Saw, Reinforced concrete, Cooling media, Wear characteristics

Posted Date: May 18th, 2021

DOI: https://doi.org/10.21203/rs.3.rs-502103/v1

License: (1) This work is licensed under a Creative Commons Attribution 4.0 International License. Read Full License 


\section{Simulation and Experimental Research on the Cooling Medium of Diamond Single Particles and Beads Cutting Reinforced Concrete}

Lan Zhang, Yongyin Pang, Ming Liu, Liquan Wang, Xianbin Sha, Yue Xiao

1. Correspondence: lium@hrbeu.edu.cn; Tel: +86-17745149882+M.L

2. College of Mechanical and Electrical Engineering, Harbin Engineering University, Harbin 150001 China

Abstract: Diamond wire saw is the first choice for the demolition of reinforced concrete walls and is expected to work from top to bottom for a couple of weeks. However, the service life of the machine is greatly affected by dry operating conditions. What's more, the thick and high walls make the effective cooling of working diamond wire saws even harder. To tackle the cooling problem in the work, not only was the cutting heat generated during the cutting process calculated to provide a theoretical basis for the simulation and experiments, but the FEM software AdvantEdge was used to simulate the cutting process. By applying four different cooling media and comparing the temperature change of the cutting zone as well as the force of the diamond particles, the best cooling scheme was obtained. What's more, the effectiveness of the cooling medium in the simulation process was experimentally verified. In the end, microscopic wear of the diamond particles was observed by Scanning Electron Microscope to study the impact of different cooling media. The proposed cooling scheme is of major significance for the dismantling of decommissioned nuclear facilities with diamond wire saws.

Keywords: Diamond Wire Saw; Reinforced concrete; Cooling media; Wear characteristics

\section{Introduction}

Since the new century, energy demand has reached a new peak and continues to rise. There is little doubt that energy-related issues will remain the greatest challenge for the rest of the century. The nuclear energy provides clean energy for us. By now, nuclear plants have been constructed worldwide and benefit millions of people on the one side, while on the other side, the accidental potential must not be overlooked. For example, the leakage accident at the Fukushima nuclear power plant in Japan in 2011 [1]. Once an accident or decommissioning of nuclear power occurs, it needs to be dismantled to achieve sustainability. Considering that nuclear power plants generally occupy a large area and the facilities are radioactive, they cannot be demolished by directional blasting. The diamond wire saw is applicable because it can dismantle the reinforced concrete walls of nuclear facilities bit by bit, which minimizes the environmental pollution during the demolition. Due to the thick and high walls of nuclear facilities, the notches need to be deep and the working platform should be high, thus posing technical challenges to the effective cooling of diamond wire saw cutting.

To minimize the harm of decommissioned nuclear power facilities, countries around the world have invested in research on dismantling technology [2]. France used diamond automatic grinders to remove nuclear reactor 
surface materials [3]; American companies used diamond wire saws to complete the dismantling of the Troy nuclear power plant vessel shell [4], and the separation of the nuclear reactor vessel cavity of the Big Rock Nuclear Power Plant [5-6]. BD Nuclear Power Company uses diamond wire saws and other equipment to complete the cutting of the biological shield of the Dragon reactor [7]. Diamond wire saws also play an important role in the ore mining industry. Because of the unique combination of rigidity and flexibility, high efficiency, and low noise, the beaded rope will be more and more widely used in the cutting field [8]. At present, a diamond beaded rope is considered to be one of the most reasonable ways to remove it. However, for the slender cut of reinforced concrete, the cooling method of long-distance cutting of beaded rope has not been fully studied yet. Research and comparison of different cooling methods are needed to find the best cooling solution when the beaded rope cuts reinforced concrete.

The diamond wire saw [9] is a high-speed cyclic intermittent grinding process for the containment of decommissioned nuclear power plants. Studying the temperature change and influence law of the cutting area of diamond particles is the theoretical basis for analyzing the high-speed cutting technology of bead. Material properties, machining and cutting parameters [10], and cooling methods are all important factors that affect the temperature of the cutting zone. For the removal of the containment of nuclear power plants on land, full consideration must be given to the setting of cooling conditions to avoid excessively high temperature in the cutting area of the tool causing carbonization of diamond particles [11]; at the same time, the cooling medium can take away the chips and prevent their accumulation from causing bead wear and reduction. Containment removal efficiency. In the process of cutting reinforced concrete with diamond beads, a large amount of cutting heat will be generated, which has a great impact on the service life of the beads [12]. To remove the reinforced concrete wall block by block [13], some scholars proposed to use cold water to directly cool the temperature, but the cold water lacks the lubricating ability and has limited thermal conductivity. In this way, the beads will still wear severely under the cooling method, and the service life of the particles will not be long. Since 1960, scholars have begun to study quasi-dry cutting between dry cutting and wet cutting. This cooling method was later used in many fields to meet basic cooling requirements. With the continuous improvement of working conditions and technical requirements, new cooling technologies have been developed and utilized, such as low-temperature cold air cooling, liquid nitrogen cooling, low-temperature spray cooling, MQL technology, etc. [14]. Ucun, I [15] et al. conducted experiments on diamond saw blades cutting natural gemstones using liquid soap as the coolant. The research showed that the surface of the workpiece with the coolant smoother. Hong et al. [16] injected a cooling medium (liquid nitrogen) into the cutting area through a micro nozzle, and successfully improved the service life 
of the tool. Qian PF[17] et al. conducted numerical simulation on low-temperature water jet cooling through Ansysfluent, and the study found that when the diameter of the water flow is greater than $4 \mathrm{~mm}$, a better cooling effect can be achieved. The American scholar S.Y. Hong [18] conducted theoretical and experimental research on liquid nitrogen cooling technology and introduced it to the grinding process of cooling. Zhou ZM [19] used the processing technology of water and vegetable oil atomization as coolant and applied it to diamond-cutting titanium alloy processing, which has achieved certain results. Pershin, G.D [20] studied the feasibility of cutting marble blocks with a diamond wire saw underwater cooling through a pilot industrial study in a quarry. The choice of the cooling medium has a great influence on the cutting performance of the diamond wire saw.

In the previous research on cutting cooling methods, most of the cutting objects are small and the cooling medium can easily reach the cutting position, which is not suitable for demolition tasks of reinforced concrete walls that focus on cutting efficiency and have huge cutting objects. To penetrate the thick reinforced concrete wall, the notch has to be deep, which requires the adaptability of the cooling medium. The work is targeted at the removal of reinforced concrete from nuclear facilities with diamond beaded ropes, the theoretical analysis of cutting heat, and theoretical support for the simulation of cutting. In the work, AdvantEdge software was used to simulate the cutting research and different cooling media was applied, the dry cutting conditions and the action of four different cooling media were analyzed, the temperature change of the diamond cutting temperature field, and the cutting force of the diamond, determine the most suitable bead rope cutting reinforced concrete Cooling medium. Based on simulation analysis, three kinds of beaded rope cutting reinforced concrete experiments under different cooling effects were carried out to verify the reliability of the simulation results. In the experiment, the performance of the effect of different cooling media is evaluated by measuring the temperature field of the cutting area and the direct wear of the beads. The following conclusions could be drawn through simulation and experimental research: Under effective cooling, not only is the temperature of the cutting area reduced, but also the integrity of the particles is improved. Among them, the effect of a low-temperature steam cooling medium is the best. The optimal cooling scheme for the diamond wire saw machine dismantling the abandoned nuclear power plant was obtained.

\section{Theoretical analysis of cutting heat}

The cutting process will be accompanied by deformation and frictional heat generation. According to Fourier's law of heat conduction[21], the heat flux is proportional to the temperature gradient. In three-dimensional heat conduction, as shown in Fig.1, the temperature gradient can be expressed as:

$$
\frac{\partial T}{\partial n}=\frac{\partial T}{\partial x} \cos a+\frac{\partial T}{\partial y} \cos \beta+\frac{\partial T}{\partial z} \cos \gamma
$$


The heat transferred to the entire surface $\mathrm{S}$ per unit time is:

$$
Q=\iiint_{V} \lambda\left[\frac{\partial}{\partial x}\left(\frac{\partial_{T}}{\partial x}\right)+\frac{\partial}{\partial y}\left(\frac{\partial_{T}}{\partial y}\right)+\frac{\partial}{\partial z}\left(\frac{\partial_{T}}{\partial z}\right)\right] d V
$$

Volume is an optional volume unit in the workpiece, the value is zero when integrated, and the three-dimensional differential equation is obtained by combining[22]:

$$
\frac{\partial T}{\partial \tau}=a\left(\frac{\partial^{2} T}{\partial x^{2}}+\frac{\partial^{2} T}{\partial y^{2}}+\frac{\partial^{2} T}{\partial z^{2}}\right)
$$

where $a$ is the thermal conductivity of the thermally conductive medium
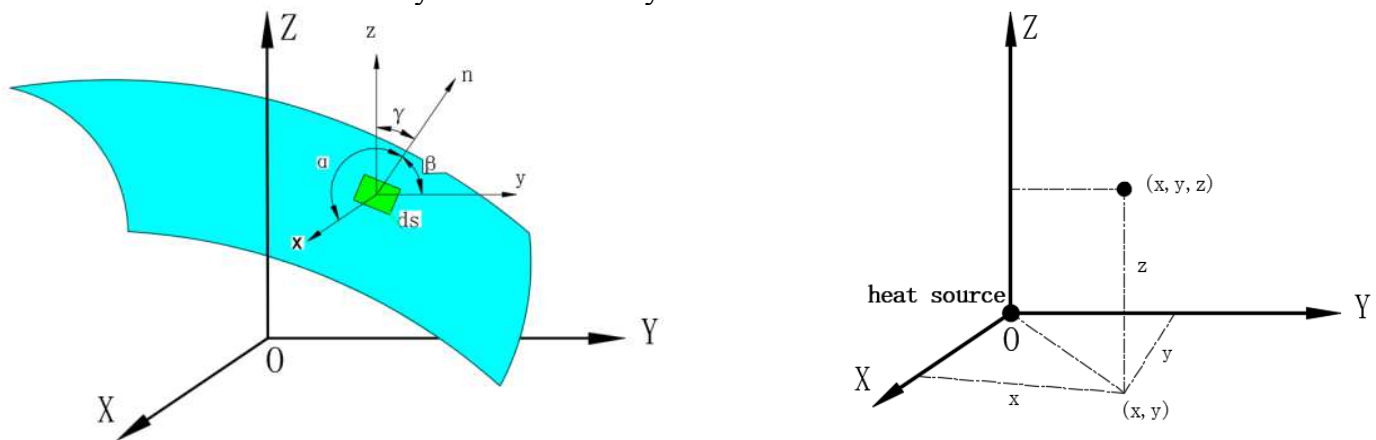

Fig. 1. Thermal differential analysis diagram. Fig.2. Coordinate model of heat source temperature field.

Assuming that the heat source point is at $(0,0,0)$, as shown in Fig.2. The corresponding temperature field formula is:

$$
T=\frac{Q_{0}}{c \rho(4 \pi a \tau)^{3 / 2}} e^{\frac{x^{2}+y^{2}+z^{2}}{4 a \tau}}
$$

where c is heat capacity, $\rho$ is density, $a$ is thermal conductivity of the thermally conductive medium.

The reasonable design of tool angles and boundaries is important in the cutting process for heat generation and heat dissipation[23]. When diamond cutting, the cutting edge is a line rather than a point, and each temperature point can be approximated as a linear heat source. Through the principle of superposition and integral reasoning, the formula of the tool surface temperature field can be obtained as:

$$
T=\frac{Q}{4 c \rho \pi \alpha} \int_{0}^{t} \frac{E(t-\tau)}{\tau} e^{-\frac{\left(x-v_{f t}+v_{f \tau}\right)^{2}+y^{2}}{4 \alpha \tau}}\left[\operatorname{erf}\left(\frac{z+l}{\sqrt{4 \alpha \tau}}\right)-\operatorname{erf}\left(\frac{z-l}{\sqrt{4 \alpha \tau}}\right)\right] d \tau
$$

where $\mathrm{Q}$ is the Point heat source's calorific value, $\operatorname{erf}\left(\frac{z+l}{\sqrt{4 \alpha \tau}}\right)-\operatorname{erf}\left(\frac{z-l}{\sqrt{4 \alpha \tau}}\right)$ is Error function, $V_{f}$ is heat source's moving speed.

According to formula (5), the theoretical temperature of the cutting temperature field can be calculated. When air is the medium in dry cutting, the parameters are as follows: $c=1.005 \mathrm{~kJ} / \mathrm{Kg} \cdot{ }^{\circ} \mathrm{C}, \rho=1.293 \mathrm{Kg} / \mathrm{m}^{3}$, $a=0.0267 \mathrm{~W} / \mathrm{m} \cdot \mathrm{k}$.Substituting into the formula $(5)$, the temperature of the cutting zone can be calculated as $T=1390.29^{\circ} \mathrm{C}$.

It can be seen from cutting experiments that the heat in the cutting area is not completely transferred to the tool and the workpiece, at least the cutting heat leaves the contact area with the chips [24]. This is the main reason 
that the theoretical calculation value is higher than the temperature in the cutting zone of the simulation and experiment. This section provides theoretical derivation for the cutting area, which provides a theoretical basis for the next step of diamond cutting reinforced concrete simulation and experiments.

\section{Finite element simulation}

\subsection{Finite element simulation hypothesis}

The paper used Third Wave AdvantEdge software for simulation analysis. The modeling is based on the following settings:

1.The workpiece material is $\mathrm{C} 40$ reinforced concrete parameters, isotropic, and the phase change caused by temperature was ignored.

2.Diamond is used as a tool for straight cutting and the fracture is ignored.

3. The cooling zone is added to the cutting zone, and the cooling medium fully contacts the cutting zone.

\subsection{Determination and modeling of the constitutive model of the workpiece}

Three-dimensional simulation is more realistic than two-dimensional, and the simulation cooling effect is better [25]. When cutting reinforced concrete, the surface of the workpiece is deformed, the temperature in the cutting zone rises, and some materials will undergo brittle fracture. The work uses the Johnson-cook model [26]to characterize the movement, the formula is as follows:

$$
\sigma=\left(A+B \bar{\varepsilon}^{n}\right)(1+C \ln \dot{\varepsilon})\left[1-\left(\frac{T-T_{r}}{T_{m}-T_{r}}\right)^{m}\right]
$$

where $A 、 B$ and n are yield strength, strain hardening strength, hardening constant, and are taken as $335,1410.3$ and 0035. $C$ is Strain hardening parameters and is taken as $0.035 . m$ and $n$ are Thermal softening parameters and hardening constant, are taken as 1.67 and 0.614 .

The three-dimensional geometric model established in AdvantEdge software is shown in Fig.3; the attribute parameters of the workpiece and the tool are shown in Table 1:

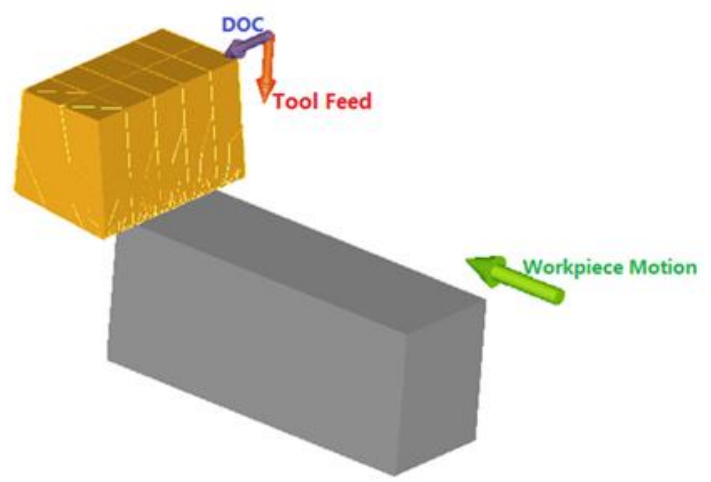

Fig. 3. Simulation three-dimensional geometric model. 
Table 1Material property parameters.

\begin{tabular}{cccccc}
\hline Material & Elastic Modulus & Poisson's ratio & Specific heat & $\begin{array}{c}\text { Thermal } \\
\text { conductivity }\end{array}$ & $\begin{array}{c}\text { Coefficient of } \\
\text { expansion }\end{array}$ \\
\hline Diamond & $1.1 \times 10^{6} \mathrm{Mpa}$ & 0.07 & $399.84 \mathrm{~J} / \mathrm{kg} .{ }^{\circ} \mathrm{C}$ & $2000 \mathrm{~W} / \mathrm{m} \cdot \mathrm{k}$ & $1.2 \times 10^{-6} \mathrm{~m} / \mathrm{k}$ \\
\hline $\mathrm{C} 40$ & $3.25 \times 10^{4} \mathrm{Mpa}$ & 0.3 & $970 \mathrm{~J} / \mathrm{kg} .{ }^{\circ} \mathrm{C}$ & $1.28 \mathrm{~W} / \mathrm{m} \cdot \mathrm{k}$ & $1.0 \times 10^{-6} \mathrm{~m} / \mathrm{k}$ \\
\hline
\end{tabular}

Combined with the actual working conditions, in the simulation design, the feed rate is set to $10 \mathrm{~mm} / \mathrm{min}$, the cutting speed is $25 \mathrm{~m} / \mathrm{s}$, the cutting length is $2.5 \mathrm{~mm}$, and the friction coefficient of the material is set as 0.4 .

In order to improve the simulation accuracy and reduce the calculation time, local meshing is used, and fine meshes are used for the contact part and adjacent parts of the blade and the workpiece [27].

\subsection{Analysis of simulation results of cutting temperature under different cooling media}

The cooling media studied in this paper are low-temperature cold air, high-pressure water, liquid nitrogen, and low-temperature steam. The low-temperature cold air cooling medium is kept below $-20^{\circ} \mathrm{C}$; the high-pressure water cooling medium is kept at $0^{\circ} \mathrm{C}$, and the liquid nitrogen cooling medium [28] is kept below $-150^{\circ} \mathrm{C}$; the lowtemperature steam is at the normal temperature. AdvantEdge is used for simulation. The action position of the cold source needs to be added within the cutting area, and the method of directly selecting the outer surface of the workpiece or the cutting surface of the tool as the cooling action surface results in a large difference between the action effect and the actual cooling effect. After research and improvement, the local selection function is applied to only add a cooling medium to the small plane of the workpiece and the cell of the tooltip. The cooling medium completely covers the cutting area, and the effect is better, as shown in Fig.4; Fig.5 is a dry Simulation cloud map of the cutting area during cutting.

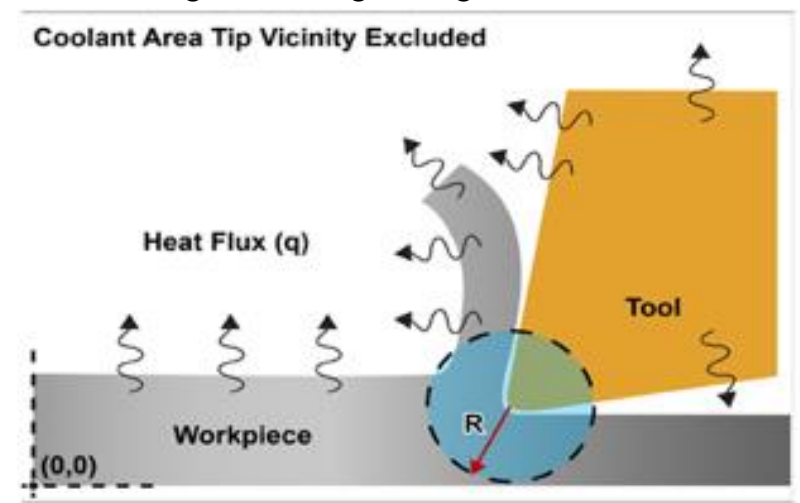

Fig.4. Application position of cold source.

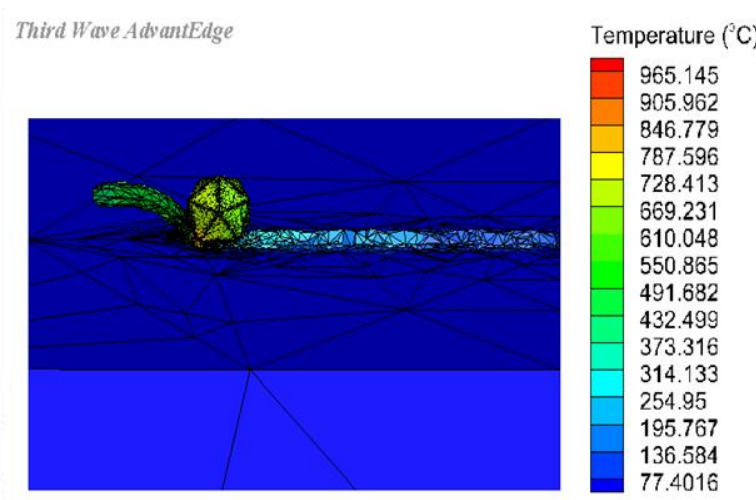

Fig.5. Temperature cloud map of cutting zone.

According to Fig.5, the highest temperature in the cutting zone under dry cutting is $965.145^{\circ} \mathrm{C}$. The maximum temperature calculated from the second section of the chip thermal theory is $1390.29^{\circ} \mathrm{C}$, which excludes about $30 \%$ of the chips carried away, and the maximum temperature in the cutting zone is about $973.203^{\circ} \mathrm{C}$. It can be seen that the simulation results are accurate and feasible. 
Based on dry cutting, other parameters are not modified to ensure that the other simulation conditions are consistent. Cooling media was added to the cutting area in sequence: low-temperature cold air, high-pressure water, liquid nitrogen, and low-temperature steam. The temperature change of the cutting zone was obtained under the action of each cooling medium and the result is shown in Fig.7.

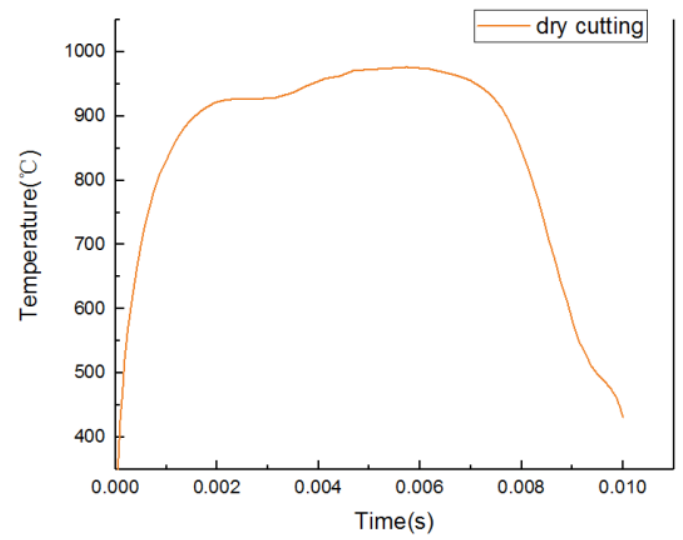

Fig.6. Dry cutting temperature curve.

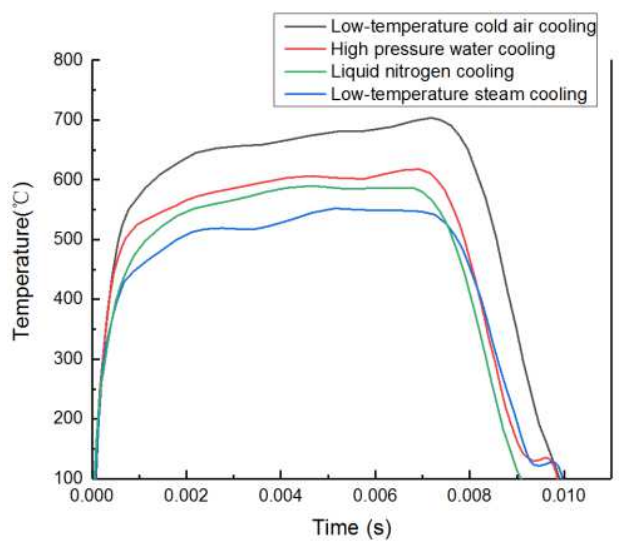

Fig.7. Temperature relationship curve diagram under different cooling media.

According to the simulation results:

(1) During dry cutting, the highest temperature in the cutting zone reaches $965^{\circ} \mathrm{C}$, which is higher than the diamond's carbonization temperature of $800^{\circ} \mathrm{C}$. The high temperature causes the diamond to be carbonized, which greatly affects the particle life.

(2) After applying the cooling medium, the highest temperature of each cutting zone is lower than $700^{\circ} \mathrm{C}$, which effectively avoids particle carbonization.

(3) Comparing the simulation results, it can be seen that the cooling effect of low-temperature spray cooling is the best. The effects of the other groups in descending order are liquid nitrogen cooling, high-pressure water cooling, and low-temperature cold air cooling.

\subsection{Analysis of simulation results of cutting force under different cooling methods}

Pressure field results of the simulation model are helpful in analyzing the pressure distribution on the workpiece. The maximum pressure is distributed in the second deformation zone, which is the contact part between the cutting edge and the workpiece during cutting, as is shown in Fig.8. This is in line with the theoretical mechanism of the cutting model. 


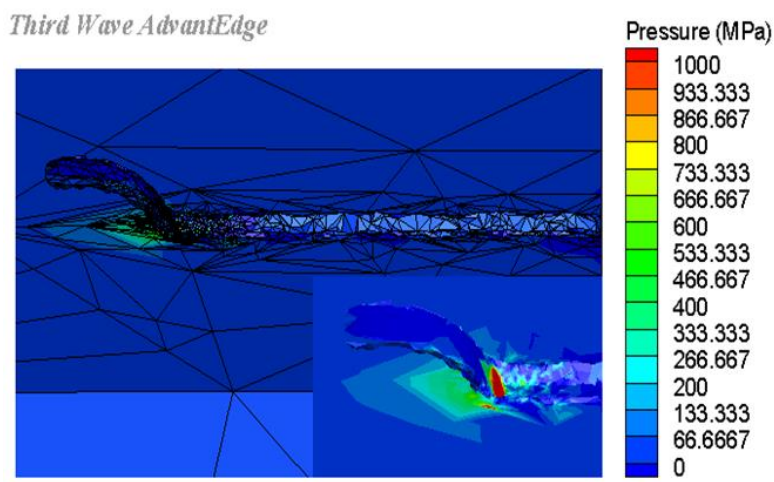

Fig.8. Three-dimensional cloud map of pressure field distribution.

Through the study of the magnitude of the force, the force of the diamond particles can be obtained under different cooling methods. The work analyzed the forces in the X, Y, and Z directions, clarified the cutting forces in each cooling mode, and provided a cooling scheme for the actual removal process.

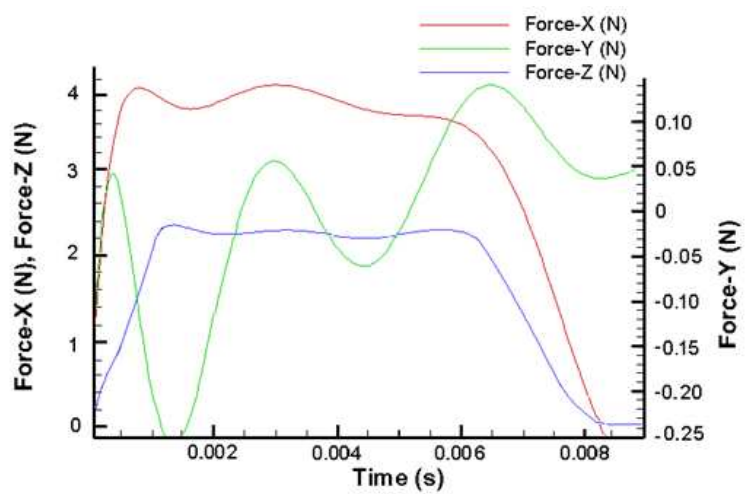

Fig.9. Dry cutting cutting force curve.

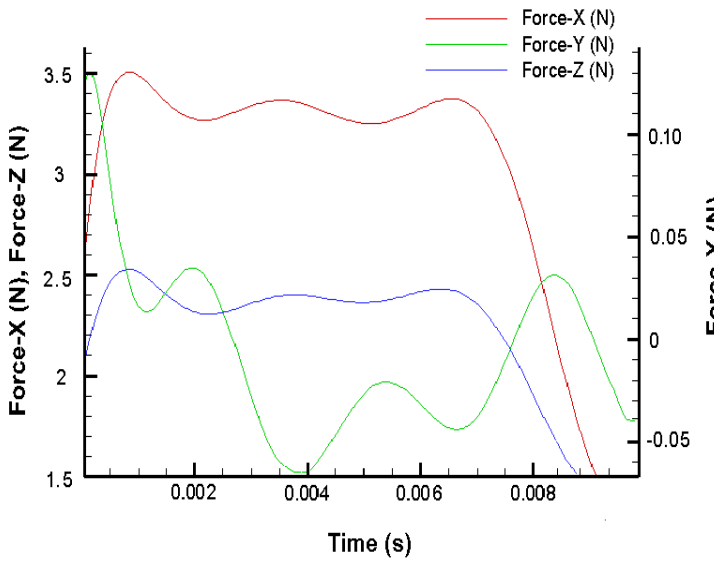

a. Low-temperature cold air cooling.

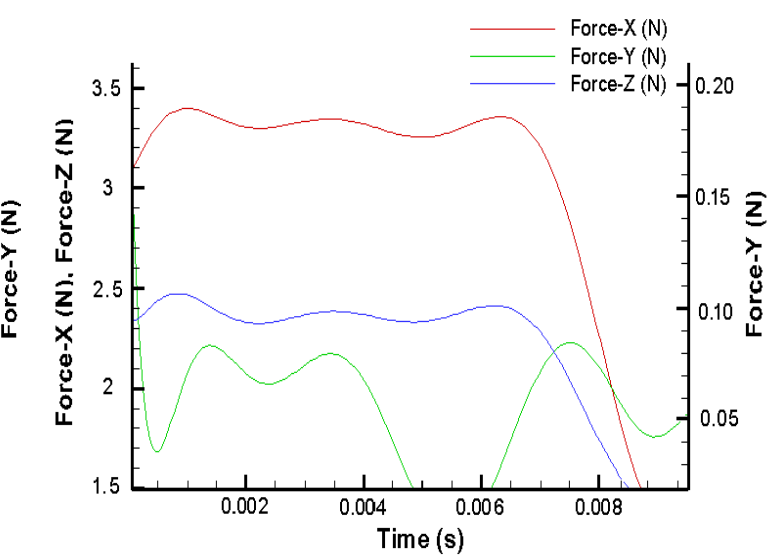

b. High-pressure water cooling. 


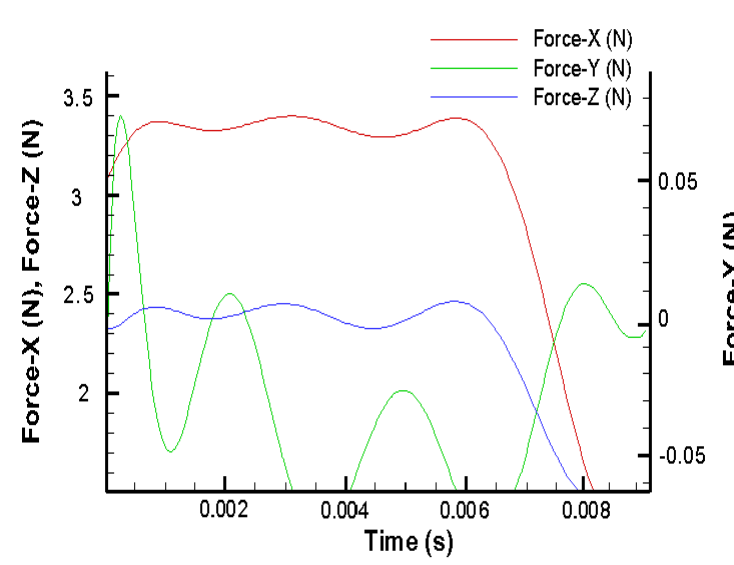

c. Liquid nitrogen cooling.

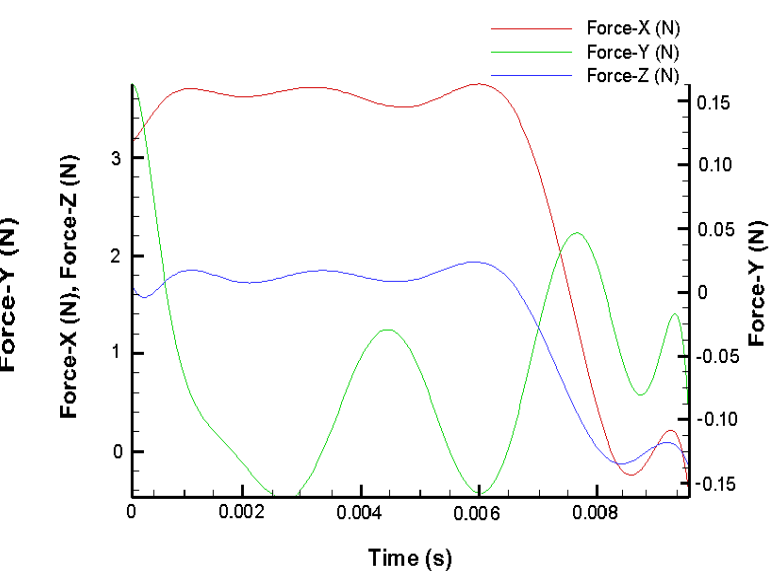

d. Low-temperature steam cooling.

Fig.10. Cutting force curve diagram under cooling medium.

Table 2 Maximum cutting force data table in each direction.

\begin{tabular}{cccc} 
cooling method & $F_{x}(\mathrm{~N})$ & $F_{y}(\mathrm{~N})$ & $F_{z}(\mathrm{~N})$ \\
\hline Dry cutting & 4.1 & 0.14 & 2.5 \\
\hline Low-temperature cold air cooling & 3.6 & 0.05 & 2.5 \\
\hline High-pressure water cooling & 3.5 & 0.08 & 2.4 \\
\hline Liquid nitrogen cooling & 3.4 & 0.02 & 2.3 \\
\hline Low-temperature steam cooling & 3.5 & 0.005 & 2.3 \\
\hline
\end{tabular}

During the cutting process, the particles are subjected to forces in three directions, of which the X-direction receives the greatest force and the main cutting force is plotted in Fig.9 and Fig.10. Table 2 shows that after applying a cooling medium, the maximum force in the $\mathrm{X}$ direction of the particles is less than the maximum cutting force during dry cutting $(4.1 \mathrm{~N})$. Moreover, the cutting force of low-temperature steam is the smallest, indicating that the cooling medium can not only reduce the temperature of the cutting zone but also reduce the force on the particles. The comprehensive effect of low-temperature steam is the best.

\section{Experiment set-up}

Simulation and performance analysis of the temperature in the cutting zone and the force on the particles were carried out, and on this basis, the cutting experiment of the beaded rope is completed. By observing the temperature in the cutting zone and the changes in the diameter of the beads, the effects of different cooling methods are analyzed to verify the accuracy of the simulation. Single-particle simulation and the use of complete objects for experimental verification are feasible [29].

The preparation of the instrument is the first step of the experiment. Experiment of cutting reinforced concrete with beaded rope was carried out on a diamond wire saw. The diamond beaded rope used in the experiment is 
produced by Jiangxi CAHARD Company, the model is: GD-11; the bead specification on the beaded rope is $\Phi 11.5$, the number of beads per meter is 40 , and the abrasive grain size is $40 \sim 50$. The bead fixing method is rubber and spring coordination, the bead is sintered, the connection is connected by a steel sleeve, and the rope length is 2.95 meters.

The reinforced concrete workpiece's size cut in the experiment is $100 \times 300 \times 500 \mathrm{~mm}$, which is composed of C40 concrete and a steel bar with a diameter of $10 \mathrm{~mm}$. The specific components are shown in Table 3 below.

Table 3 Workpiece material composition and performance parameters.

\begin{tabular}{cccc} 
material & model & ingredient & Compressive strength (Mpa) \\
\hline Grade 1 steel bar & Q235 & Iron、C、MN、si & 235 \\
\hline Concrete & C40 & Cement 、sand gravel & 40 \\
\hline
\end{tabular}

The three sets of experiments in this paper are dry cutting, low-temperature steam cooling, and high-pressure water cooling cutting. Among them, the low-temperature steam method is to use a nozzle to align the pressurized low-temperature water with the cutting area and spray it. Considering the large volume of the workpiece, multiple cooling medium spray heads are set to ensure that the cooling medium completely covers the cutting area as in the simulation analysis. The instrument and experimental results are shown in Fig. 11:
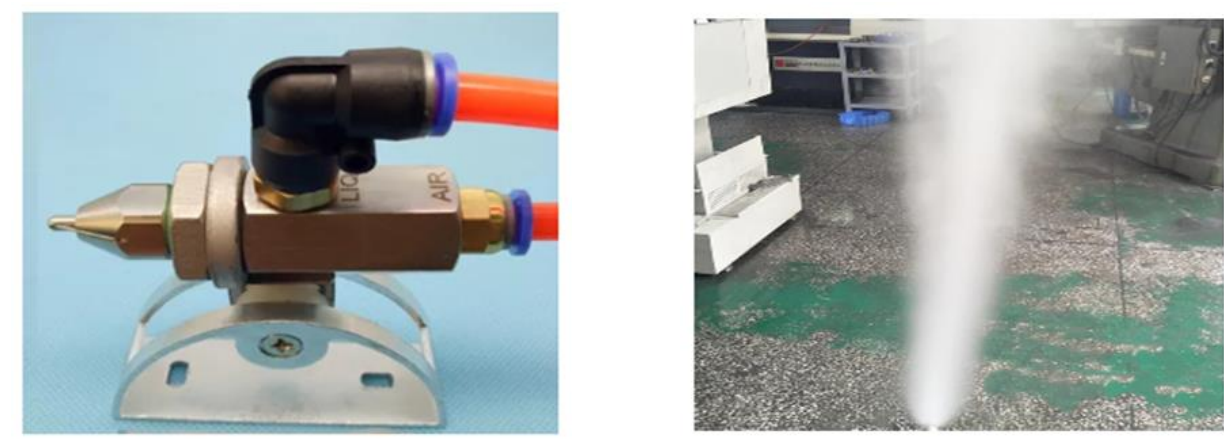

Fig.11. Low-temperature water atomization nozzle and atomization effect.

In the experiment, a temperature sensor was used to measure the temperature of the cutting area. In order to obtain more accurate experimental data and avoid the distortion of the experiment caused by temperature diffusion, the sensor was inserted into the cut position of the cutting area in this experiment. The outer micrometer and the scanning electron microscope were used to measure the change in the diameter of the beads and the change in the integrity of the particles in the experiment to study the worn form of the diamond particles under various working conditions. The equipment is shown in Fig.12. 


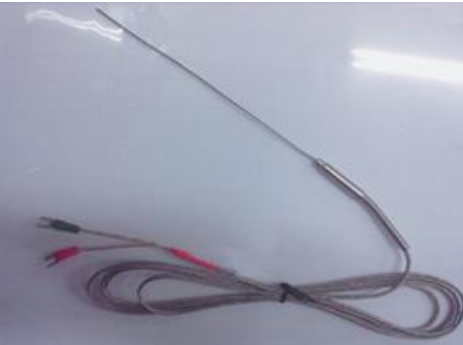

a. Thermocouple temperature sensor.

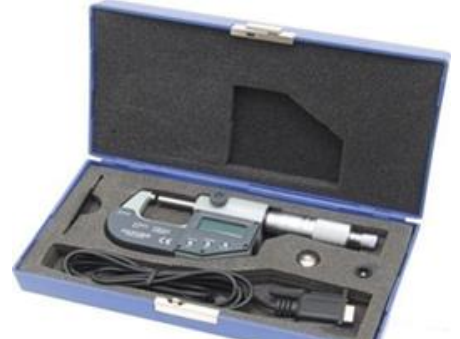

b. Outside micrometer.

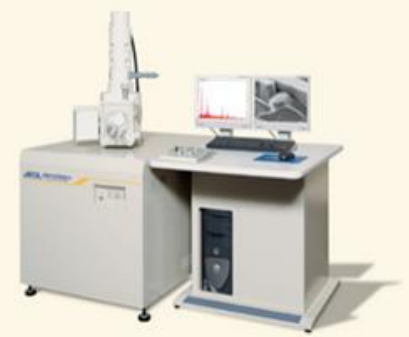

c. scanning electron microscope.

Fig.12. Experimental measuring equipment.

In the end, the experimental platform needs to be built. Experimental platform consists of the wire saw machine, temperature sensor, and cooling device selected in the previous section. Through the sensor, the temperature of the cutting zone can be monitored. Microscope etc. measures the change of beading at each stage.

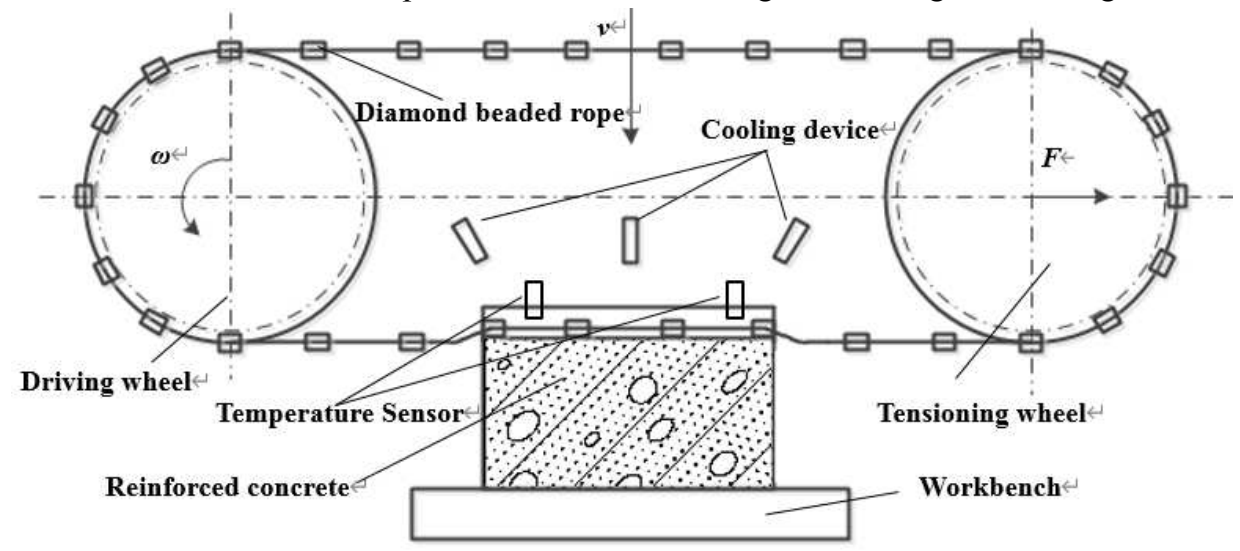

Fig.13. Schematic diagram of the experimental platform.

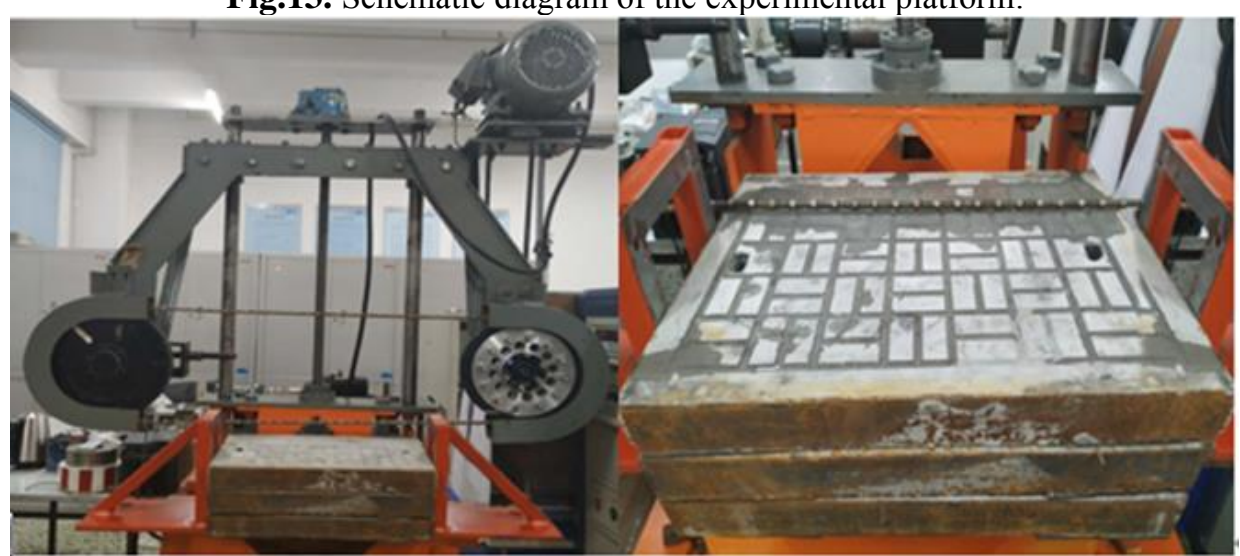

Fig.14. Experiment platform.

As shown in Fig. 14, the reinforced concrete is clamped on the platform to simulate the actual cutting conditions. Adjust the tension of the beaded rope so that it is in full contact with the concrete. Set the beaded rope cutting speed to $25 \mathrm{~m} / \mathrm{s}$ and the feed speed to $12 \mathrm{~mm} / \mathrm{min}$.

\subsection{Experimental results and analysis}

\subsubsection{The influence of cooling medium on cutting temperature}

The reinforced concrete was cut with beaded rope to study its temperature and wear under three cutting conditions. During the experiment, the thermocouple temperature sensor feedback obtained the cutting zone 
temperature under dry cutting, low-temperature spray cooling, and high-pressure water cooling. As is shown in Table 4.

Table 4 Measuring temperature of cutting zone and simulation thermometer.

\begin{tabular}{ccc}
\multirow{2}{*}{ cooling method } & \multicolumn{2}{c}{ Maximum temperature of the cutting zone $\left({ }^{\circ} \mathrm{C}\right)$} \\
\cline { 2 - 3 } & Simulation results & Experimental results \\
\hline Dry cutting & 880 & 847 \\
\hline High-pressure water cooling & 610 & 585 \\
\hline Low-temperature steam cooling & 560 & 532 \\
\hline
\end{tabular}

According to the comparative analysis in Table 4, the maximum temperature of actual cutting is slightly smaller than the maximum temperature of the simulation analysis, but the temperature changes under different cooling methods are consistent, indicating that the simulation results are reliable and can provide a theoretical basis for the removal of reinforced concrete. In the simulation and experiment, the temperature of the cutting zone in dry cutting is much higher than the diamond carbonization temperature $\left(680^{\circ} \mathrm{C}\right)$. It is necessary to set an effective and reasonable cooling method. Compared with the other two working conditions, the cooling effect of lowtemperature steam is the most obvious, which can greatly reduce the temperature of the cutting zone.

The main reason why the maximum temperature value measured in the experiment is slightly smaller than the simulation value is that in the simulation study, the finite element method is used to feedback the temperature value of any point in the cutting zone in real-time; but in the experiment, the temperature feedback by the temperature sensor is thermoelectric. The highest temperature value of the cutting zone surface that even touches can not provide real-time feedback of the cutting temperature to all nodes, so there was deviation.

\subsubsection{Influence of Cooling Medium on Wear Characteristics of Diamond Single Particles}

During the cooling experiment, a total of 8 beads are taken from the top, tail, and middle section of the beaded rope as the test objects for macroscopic and microscopic measurements. First, measure the initial outer diameter of the beads selected in each group of experiments. After the experiment, perform another measurement at the place where the wear is greatest, that is, the smallest outer diameter of the bead. Each outer diameter measurement is performed 3 times in the circumferential direction, and the result is the smallest measurement value. The 8 groups of data of the same experimental group were averaged and sorted into the bead diameter change table shown in Table 5. 
Table 5 The change of the average diameter of the beads under different cooling methods.

\begin{tabular}{cccc} 
Experimental Study & \multicolumn{3}{c}{ Bead diameter $(\mathrm{mm})$} \\
\hline Cutting time(min) & Dry cutting & High-pressure water cooling & Low-temperature steam cooling \\
\hline 0 & 11.5625 & 11.5353 & 11.5492 \\
\hline 15 & 11.4916 & 11.4899 & 11.4899 \\
\hline 30 & 11.4319 & 11.4472 & 11.4472 \\
\hline 45 & 11.3993 & 11.4529 & 11.4259 \\
\hline 60 & 11.3558 & 11.3937 & 11.3937 \\
\hline 70 & 11.3344 & 11.3838 & 11.3835 \\
\hline
\end{tabular}

And draw the graph in Fig. 15 based on the measurement data in Table 5.

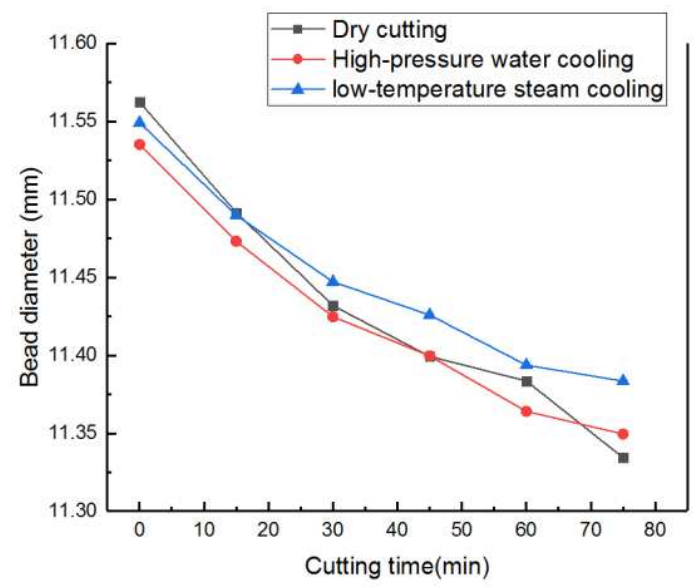

Fig.15. Variation curve of bead diameter under different cooling media.

From Fig.15, The dry cutting group with the highest cutting temperature has the largest curve slope, which shows that the outer diameter of the bead in this group has the fastest change and the most severe wear. Compared with dry cutting, the change rate of the outer diameter of the beads in the experimental group with the cooling mode is much lower than that of the dry cutting group, and the curve of low-temperature steam cooling is generally smoother and the wear rate is slower than that of high-pressure water cooling.

In the experiment, with the assistance of a scanning electron microscope, the wear degree of the diamond particles on the outer surface of each group of 8 beads was counted, the data was recorded and the average percentage was taken to form a pie chart as shown in Fig.16.
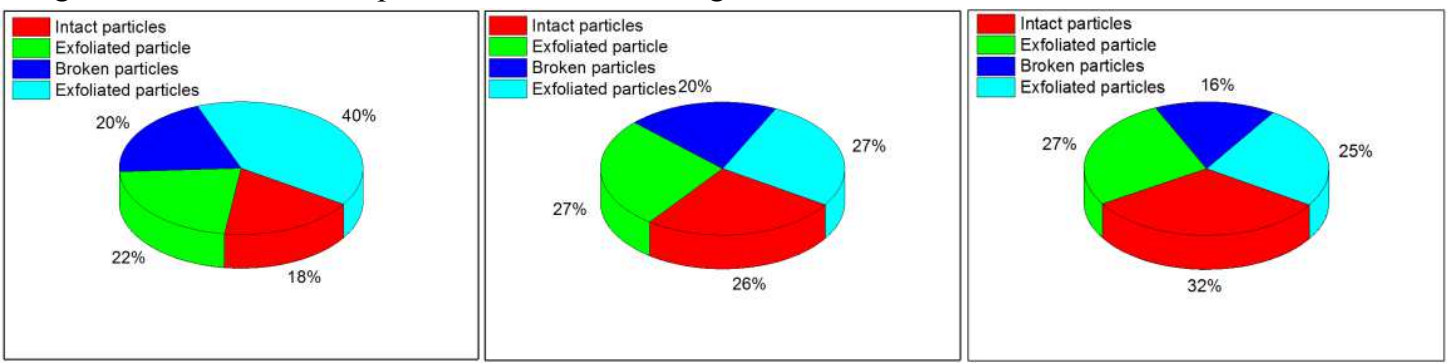
a. Dry cutting. $\quad$ b. High-pressure water cooling. c. Low-temperature steam cooling.

Fig.16. The distribution ratio of diamond particles wear state under different cooling media.

As shown in Fig.16, the proportion of particles falling off during dry cutting reaches $40 \%$ of the total wear particles; it is the main form of particle failure. After applying the cooling medium, the particle integrity ratio is greatly improved. Among them, the effect of low-temperature steam is the most obvious. The particle integrity rate reaches $32 \%$, which is $14 \%$ higher than that of dry cutting; the particles are protected to the greatest extent and the service life of the beaded rope is increased.

\section{Conclusion}

This paper completes the theoretical analysis of chip thermal when diamond cutting reinforced concrete, which provides theoretical support for simulation and experiment. AdvantEdge software was used to simulate and analyze the cutting zone temperature and the cutting force of diamond during dry cutting when cutting with four different cooling media. The bead rope cutting experiments were conducted to study the cutting zone temperature and bead wear and verify the reliability of simulation and theory. The main conclusions are listed as follows:

1) In the process of diamond cutting reinforced concrete, the higher the temperature in the cutting zone, the more serious the wear on the beads. Dry cutting will lead to particle carbonization, which will seriously affect the service life of diamond particles and reduce the efficiency of cutting operations.

2) Four different cooling media: low-temperature cold air cooling, high-pressure water cooling, liquid nitrogen cooling, and low-temperature steam cooling were simulated. The results show that the maximum temperature of each group of the cutting zone is not higher than $700^{\circ} \mathrm{C}$, which is lower than the carbonization temperature of the tool. Among them, low-temperature steam cooling features both the best cooling effect and wear resistance.

3) Through the simulation study of the cutting force of diamond particles under four different cooling media, it can be concluded that the cooling media can not only reduce the temperature of the cutting zone, but also have a lubricating effect, which can reduce the cutting force and wear of the tool, thus achieving the purpose of increasing the life of the diamond particles. Among them, the cutting force of the cutting tool under lowtemperature steam cooling is the smallest and the effect is the best.

4) Experiments show that the cooling medium can reduce the temperature of the cutting zone and slow down wear. Under the same conditions, the low-temperature steam cooling method improves the integrity of the particles by $14 \%$ compared with the dry cutting, which can maintain the integrity of the particles to the maximum and ensure efficiency. When the steam acts within certain range, low-temperature steam should be used as the cooling medium. 


\section{Acknowledgment}

Not applicable.

\section{Authors' contributions}

$\mathrm{LZ}$ conceived the research and wrote the manuscript; YP, ML and XS conducted experiments; LZ,LW and YX revised the manuscript. All authors have read and agreed to the published the version of the manuscript.

\section{Funding}

This paper is funded by the Fundamental Research Funds for the Central Universities (Grant NO.HEUCFP201848) and the National Natural Science Foundation of China (Grant NO.5167051260).

\section{Competing interests}

The authors declare that they have no competing interest.

\section{Authors' information}

Lan Zhang, currently an associate professor of Harbin Engineering University, mainly engaged in underwater intelligent operation technology and underwater special operation device and system technology.

Yongyin Pang, currently a master's student in the School of Mechanical and Electrical Engineering, Harbin Engineering University.

Ming Liu, Doctor of Engineering, currently mainly researches underwater intelligent operation technology.

Liquan Wang, born in 1957, Professor, Mainly engaged in research work in the fields of bionic robots, special operation robots, underwater production system technology, underwater operation intelligent equipment technology, and marine engineering equipment technology.

Xianbin Sha, currently a master's student in the School of Mechanical and Electrical Engineering, Harbin Engineering University.

Yue Xiao, main research interest is marine engineering technology research.

\section{Reference}

[1] Turner D.R. Nuclear Facilities, Decommissioning of Nuclear Energy[M]. Springer New York, 2013: 2-3.

[2] Sangsoo Han et al. Decontamination of concrete waste from nuclear power plant decommissioning in South Korea[J]. Annals of Nuclear Energy, 2020, 149

[3] Agency I A E. Status of the decommissioning of nuclear facilities around the world[J]. Physics Today,2004,45(9): 42-50.

[4] Cepkauskas, Martin D. Method of decommissioning a nuclear reactor[J]. Nuclear Technology,1994,74(3): 324-332.

[5] Lee W, Hirai M, Hirose S. Gunryu III: reconfigurable magnetic wall-climbing robot for decommissioning of nuclear reactor[J]. Advanced Robotics,2013,27(14): 1099-1111.

[6] Hyun D, Kim I, Lee J, et al. A methodology to simulate the cutting process for a nuclear dismantling simulation based on a digital manufacturing platform[J]. Annals of Nuclear Energy,2017,103: 369-383.

[7] Simon R. The primary circuit of the dragon high temperature reactor experiment[J]. 2005. 
[8] R.Garrard, S.R.Peacock, M.Hori. The future role of diamond in the construction industry[J]. Industrial Diamond Review,2001,61(589): 121-122.

[9] Liwen Cao, Research on cutting technology of underwater diamond wire saw,2008 IEEE International Conference on Automation and Logistics,Qingdao,2008,pp.27512756,doi:10.1109/ICAL.2008.4636641.

[10] Qi Fenglian,Lei Dongliang.Characteristic element and milling force on column diamond milling cutter during the processing stone. 2010 International Conference on Mechanic Automation and Control Engineering, Wuhan, 2010, pp. 5706-5709, doi: 10.1109/MACE.2010.

[11] Kaminski J, Alvelid B. Temperature reduction in the cutting zone in water-jet assisted turning[J]. Journal of Materials Processing Tech,2000,106(1): 68-73.

[12] Lučić Miroslav et al. Numerical Analysis of the Temperature Field in the Cutting Zone in Continuous and Discontinuous Metal Cutting by Turning[J]. Tehnički vjesnik, 2020, 27(5) : 1486-1491

[13] Cepkauskas, Martin D. Method of decommissioning a nuclear reactor[J]. Nuclear Technology,1994,74(3): 324-332.

[14] Rahman, Kumar S, Salam. Experimental evaluation on the effect of minimal quantities of lubricant in milling[J]. International Journal of Machine Tools \& Manufacture,2002, 42(5): 539-547.

[15] Ucun I , Aslantas K , Buyuksagis I , et al. Effect of cooling liquids on cutting process using diamond segmented disc of natural stones[J]. Proceedings of Institution of Mechanical Engineers Part C Journal of Mechanical Engineering Science, 2013, 227(10):2315-2327.

[16] S.Y. Hong, I. Markus, W.-C. Jeong. New cooling approach and tool life improvement in cryogenic machining of titanium alloy Ti-6Al,4V, Int. J.Machine Tools Manufacture 41 (15) (2001) 2245-2260.

[17] Qian P F, Liu Y, Gu S H, et al. Research on Cooling Technology of Shredder Cutting Tool With Ultrasonic Vibration-Assisted Cutting[J]. Ieee Access, 2019, 7: 140513-140523.

[18] S.Y.Hong. Advancement of economical cryogenic machining technology[C]. Third International Conference on Manufacturing Technology,1995: 168-173.

[19] Zhou Z, Zhang Y, Li X, et al. Application of gas-fluid atomization technology in ultrosonic vibration cutting titanium alloy workpiece[J]. Acoustical Physics, 2009, 55(6):925.

[20] Pershin G D, Gabbasov B M. Substantiation of possibility of diamond-rope cutting without water cooling[J]. Gornyi zhurnal, 2008(1): 37-40.

[21] Diane Greco,M,A.Fourier and the theory of heat[J]. Journal of Materials Processing Tech.

[22] Xu J.H, Ren K.Q, Geng G.S. Cutting forces in high-speed milling of a close alpha titanium alloy[J]. Key Engineering Materials, 2004, 259-260: 5.

[23] Cheng Hu et al. Cutting temperature prediction in negative-rake-angle machining with chamfered insert based on a modified slip-line field model[J]. International Journal of Mechanical Sciences, 2020, 167

[24] Ceretti E, Filice L, D Umbrello, et al. ALE Simulation of Orthogonal Cutting: a New Approach to Model Heat Transfer Phenomena at the Tool-Chip Interface[J]. CIRP Annals - Manufacturing Technology, 2007, 56(1):69-72

[25] Movahhedy M.R, Gadala M.S, AltintasY. Simulation of chip formation in orthogonal metal cutting process: an ale finite element approach[J]. Machining Science and Technology, 2000, 4(1): 28.

[26] Zhao C, Guan Y.F. The experiments and simulation of titanium cutting based on AdvantEdge software[J]. Applied Mechanics and Materials, 2012: 159.

[27] Erliang Liu et al. Simulation and experimental research on tool temperature field for low- temperature cutting of Ti-5553[J]. Ferroelectrics, 2020, 563(1) : 139-147.

[28] Paul S, Chattopadhyay. Determination and control of grinding zone temperature under cryogenic cooling[J]. International Journal of Machine Tools \& Manufacture, 1996, 36(4): 491-501.

[29] Bagherzadeh A, Kuram E , Budak E . Experimental evaluation of eco-friendly hybrid cooling methods in slot milling of titanium alloy[J]. Journal of Cleaner Production, 2021, 289:125817. 
Figures

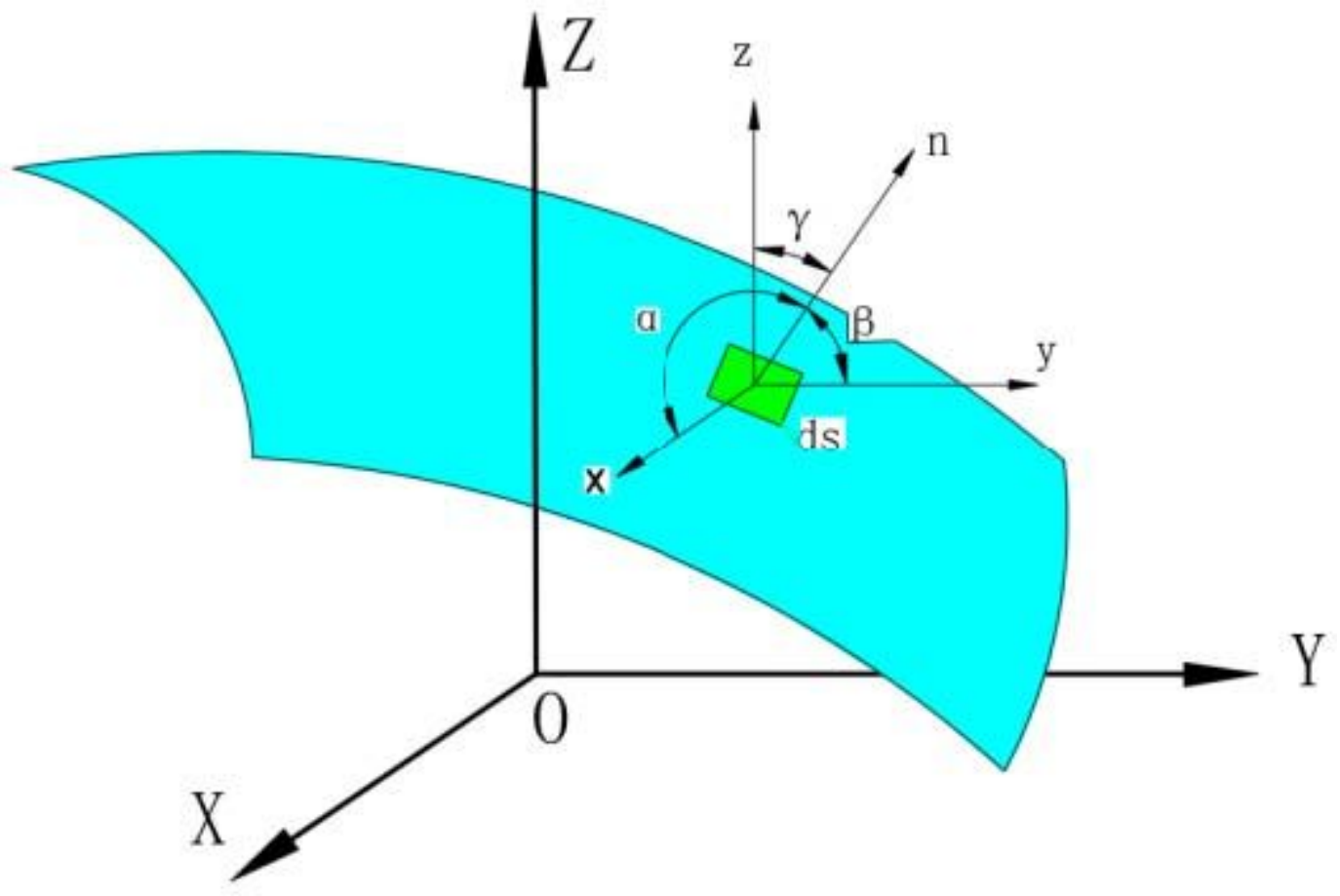

Figure 1

Thermal differential analysis diagram.

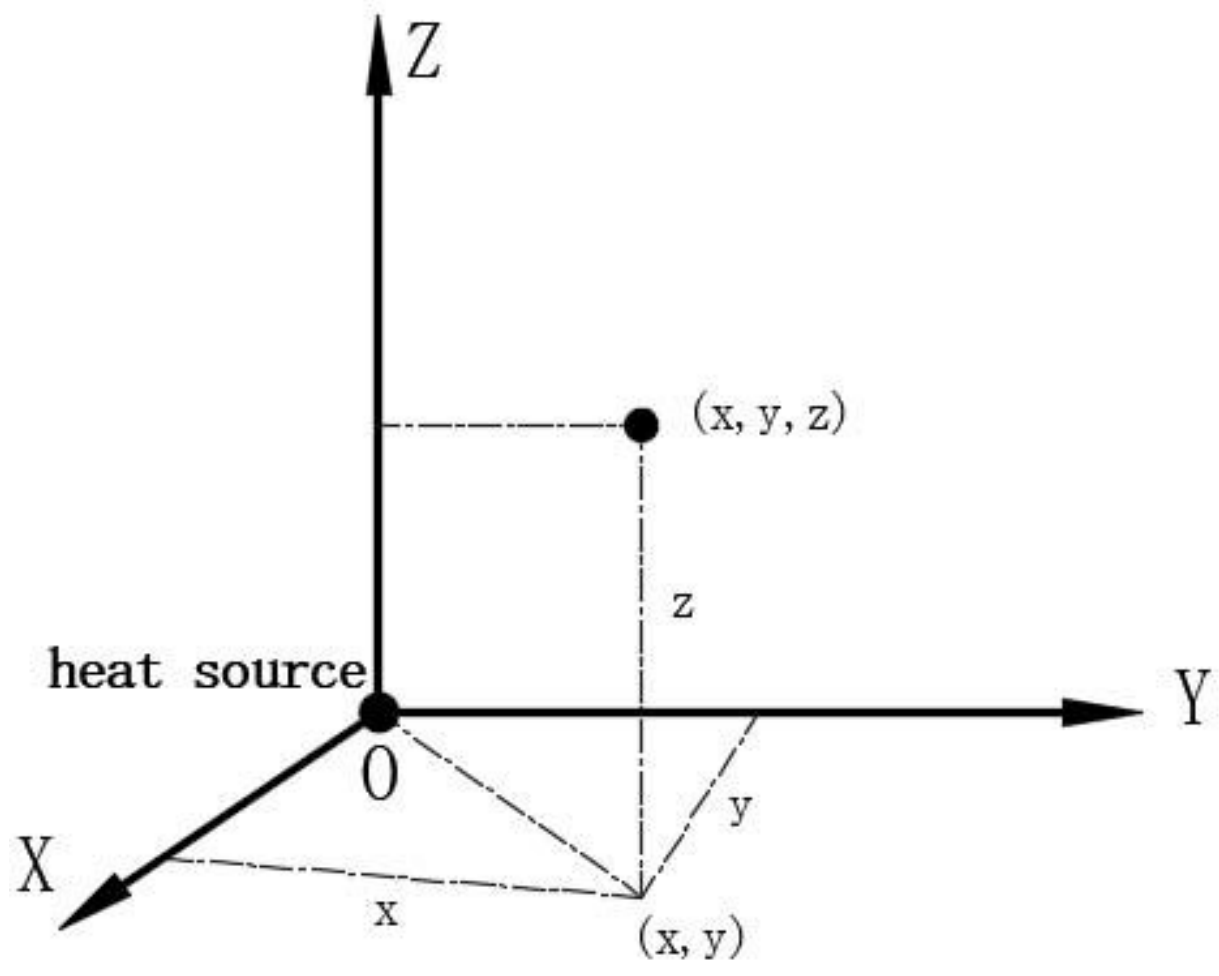

Figure 2 
Coordinate model of heat source temperature field.

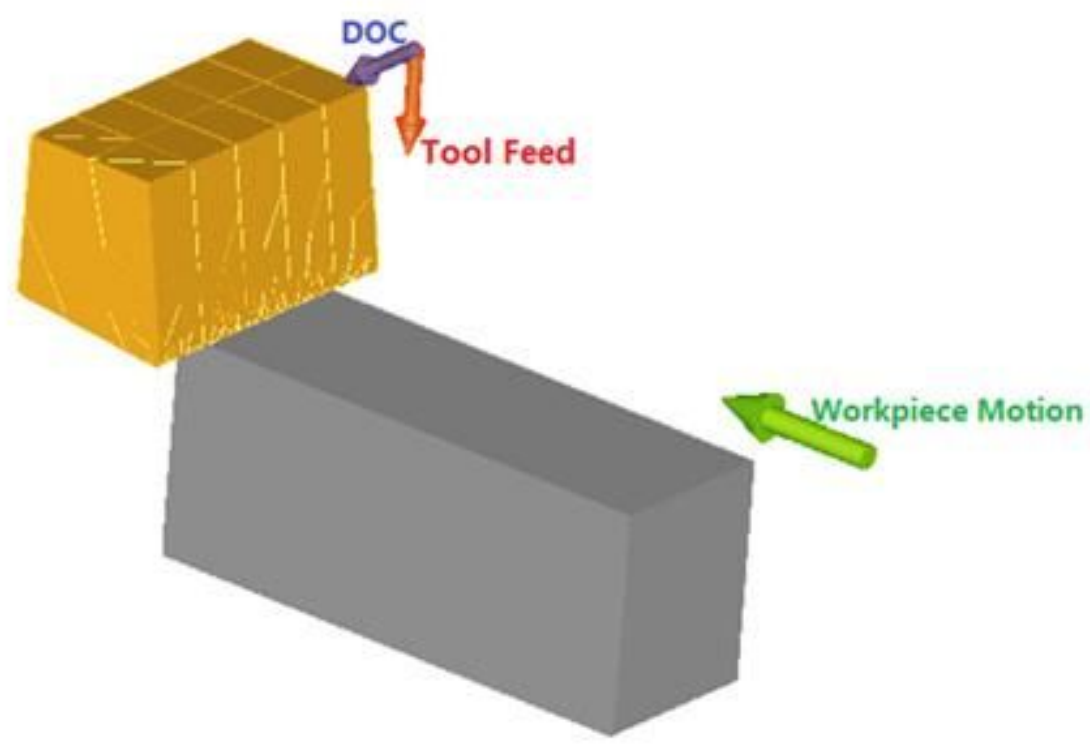

\section{Figure 3}

Simulation three-dimensional geometric model.

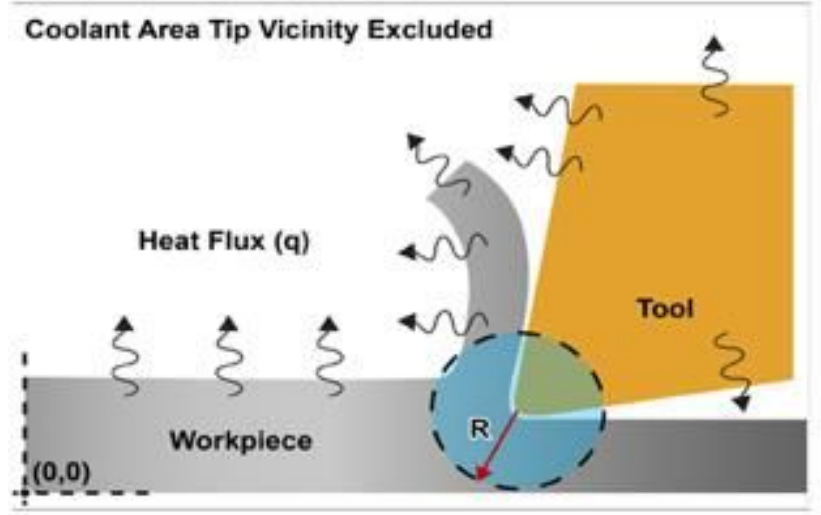

Figure 4

Application position of cold source. 

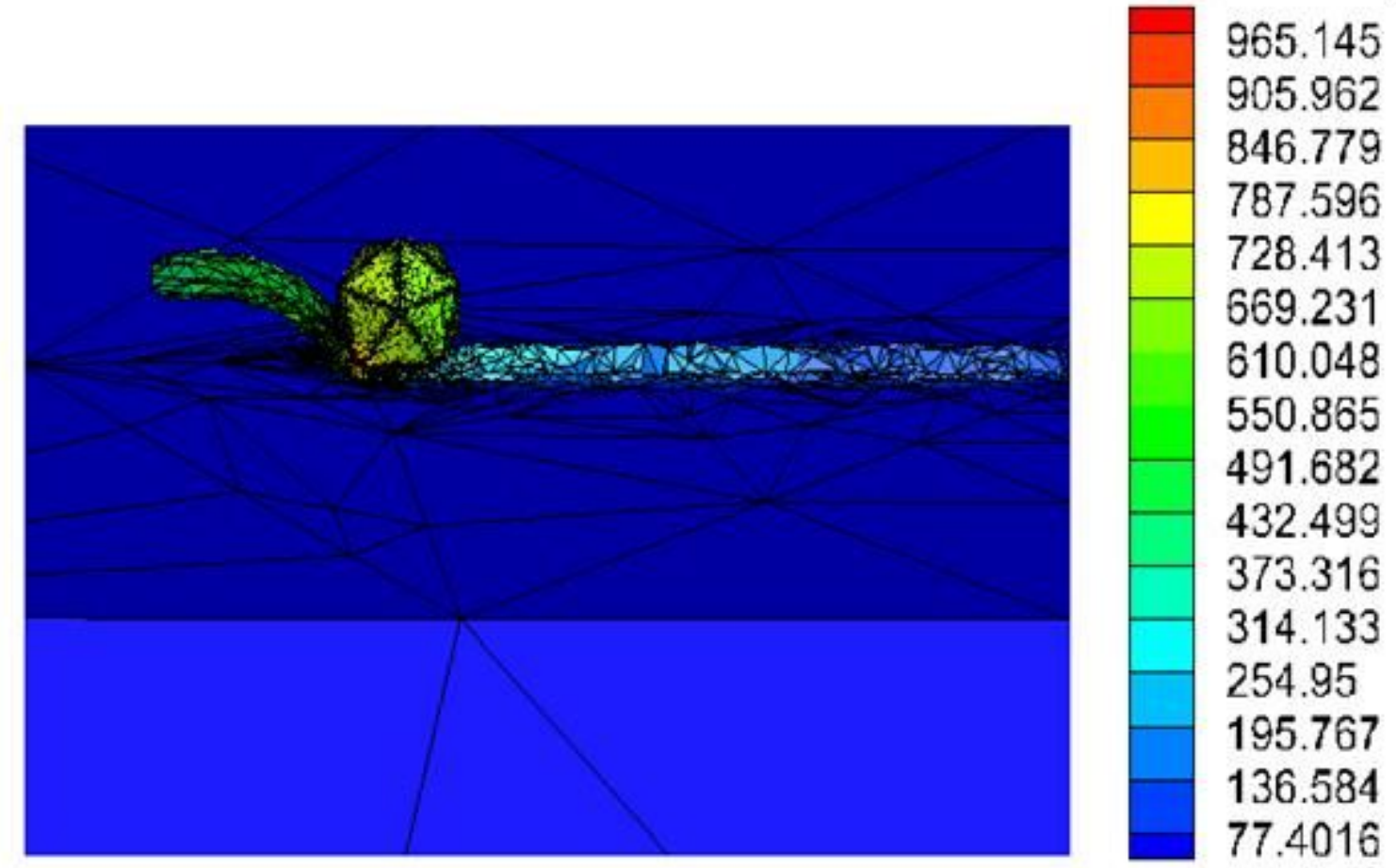

Figure 5

Temperature cloud map of cutting zone.

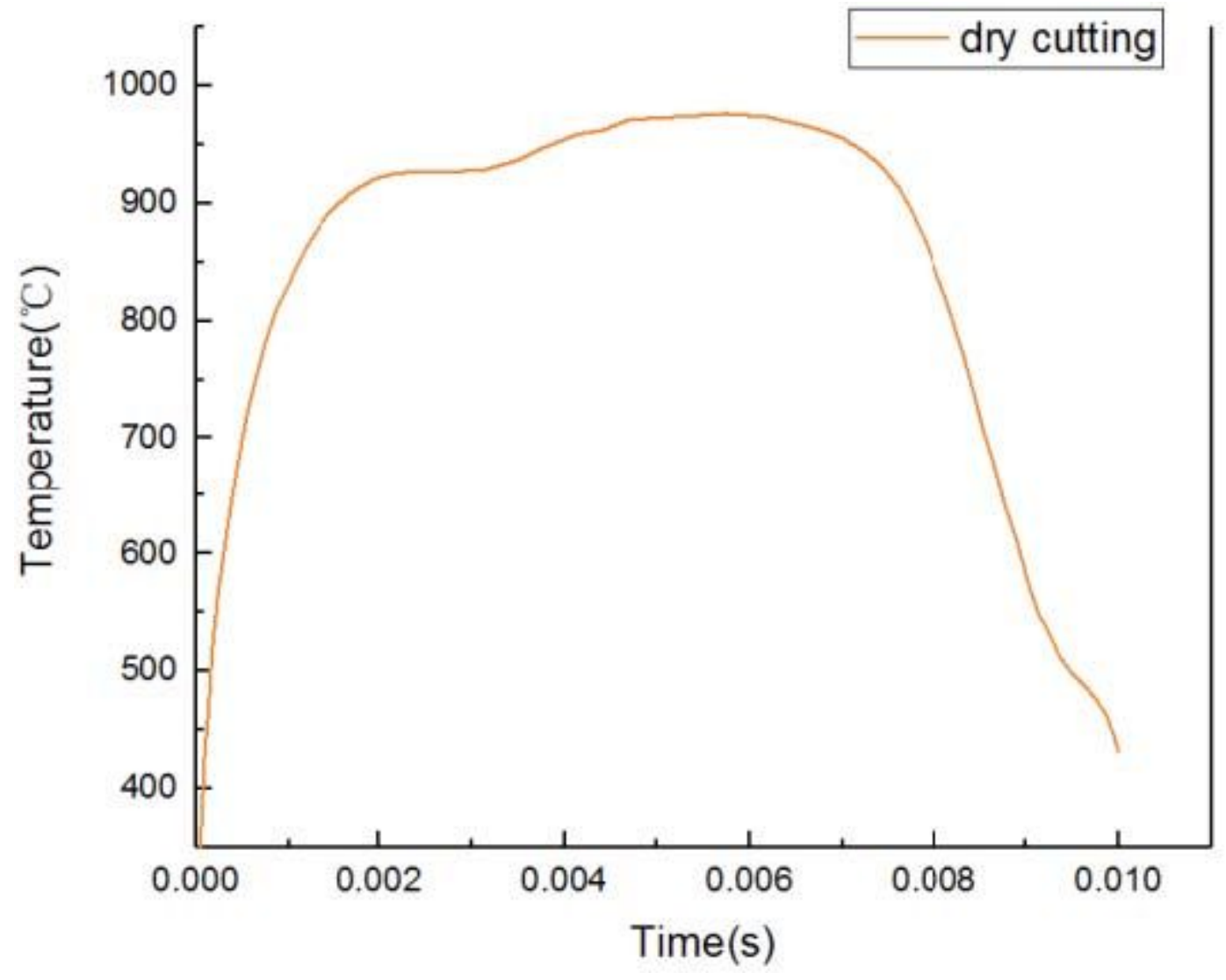

Figure 6 
Dry cutting temperature curve.

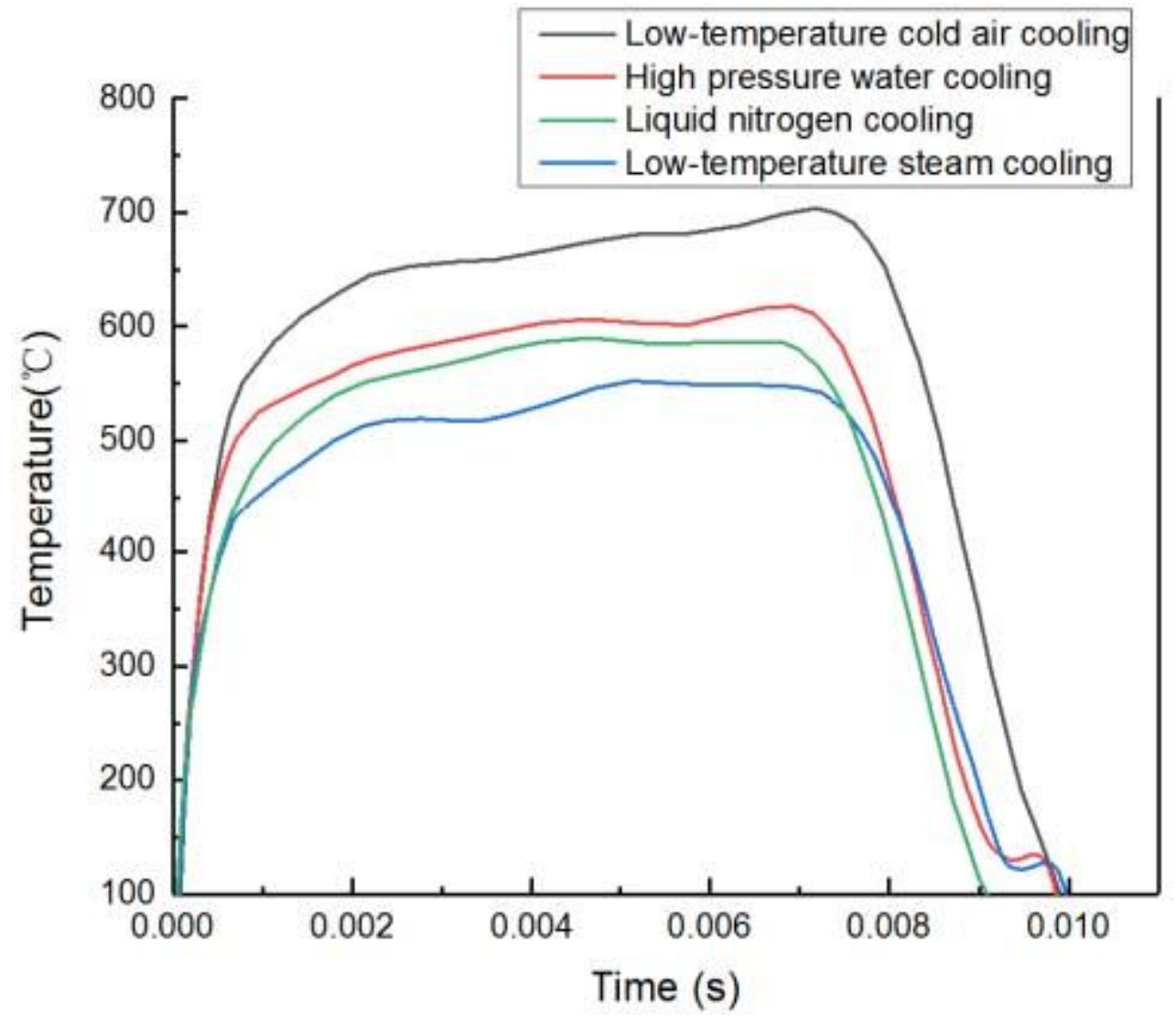

Figure 7

Temperature relationship curve diagram under different cooling media.

\section{Third Wave AdvantEdge}

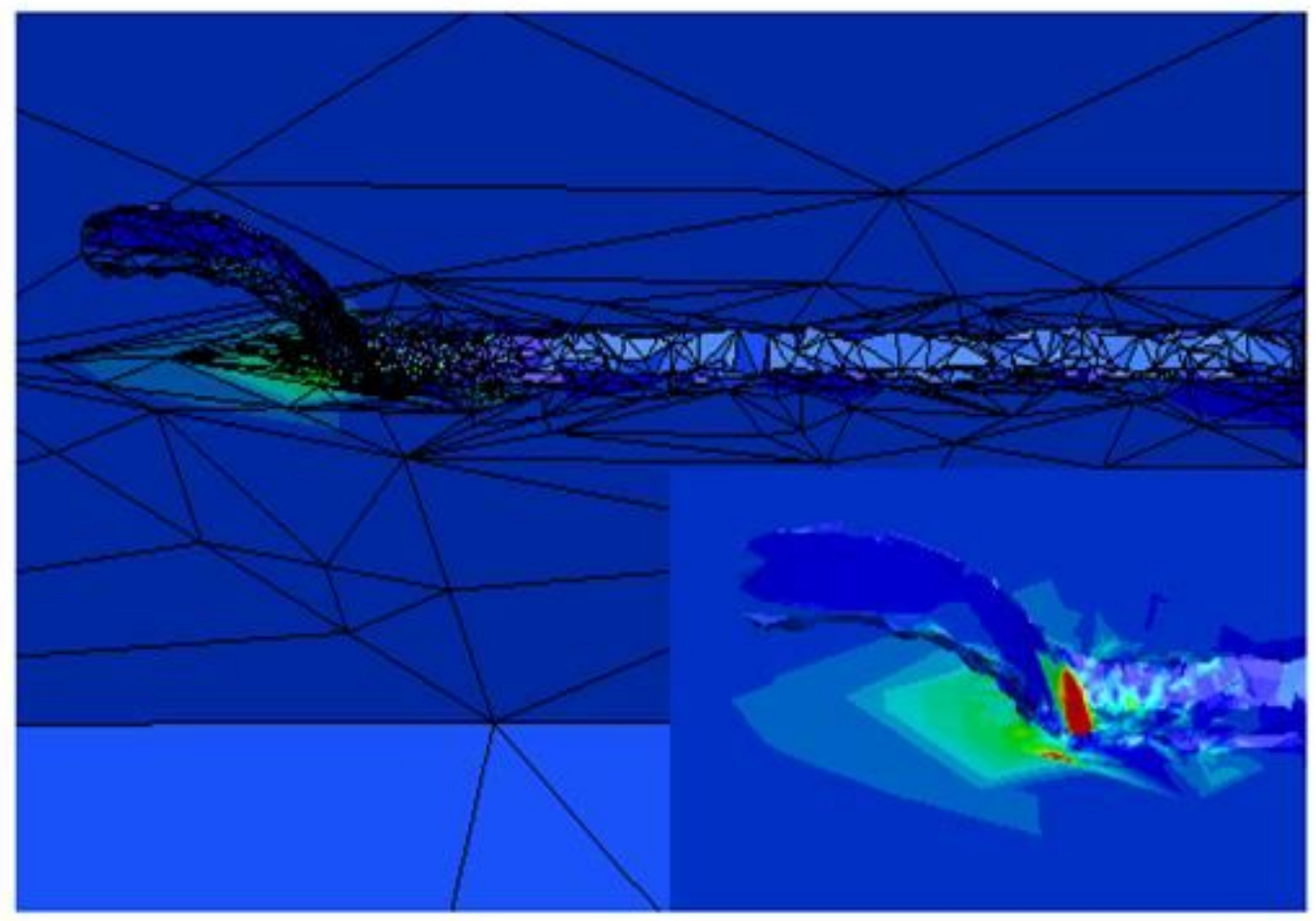

Pressure (MPa)

1000

933.333

866.667

800

733.333

666.667

600

533.333

466.667

400

333.333

266.667

200

133.333

66.6667 
Figure 8

Three-dimensional cloud map of pressure field distribution.

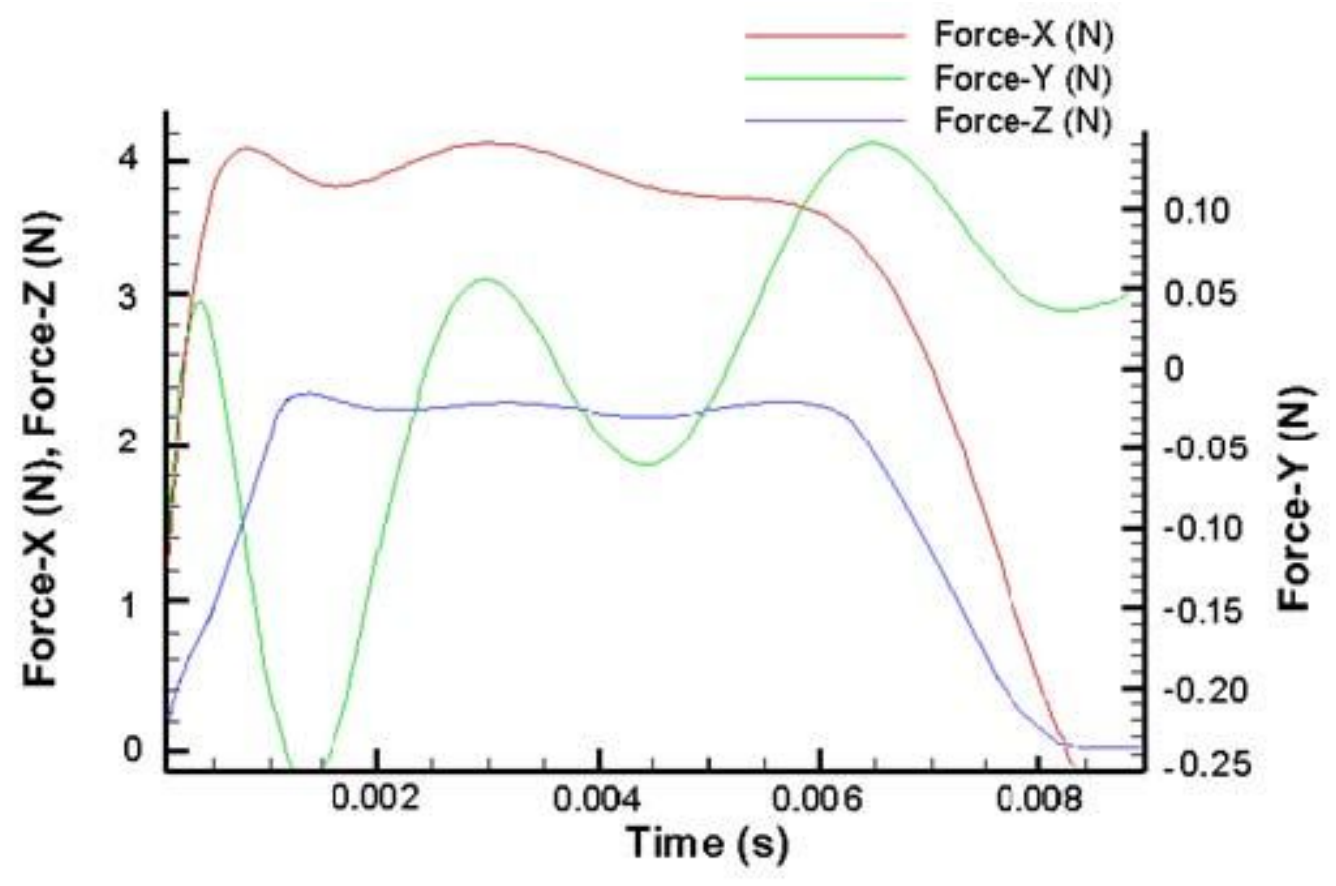

Figure 9

Dry cutting cutting force curve. 


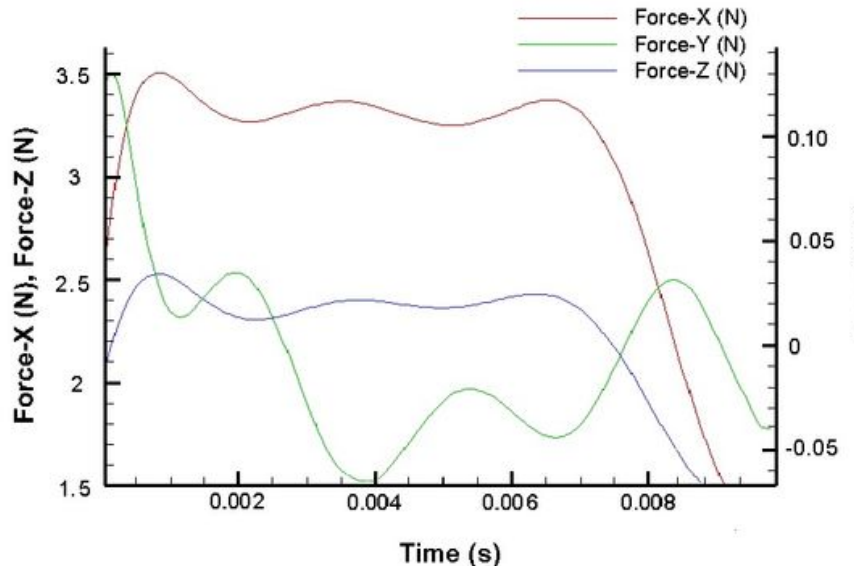

a. Low-temperature cold air cooling.

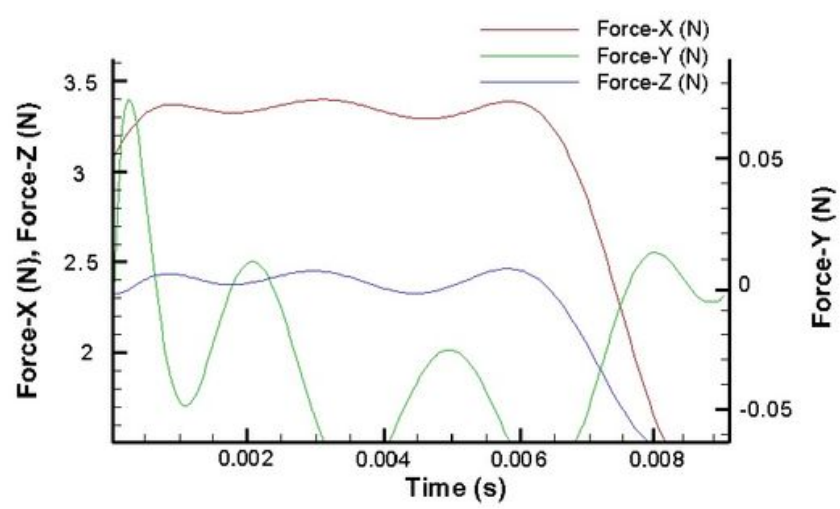

c. Liquid nitrogen cooling.

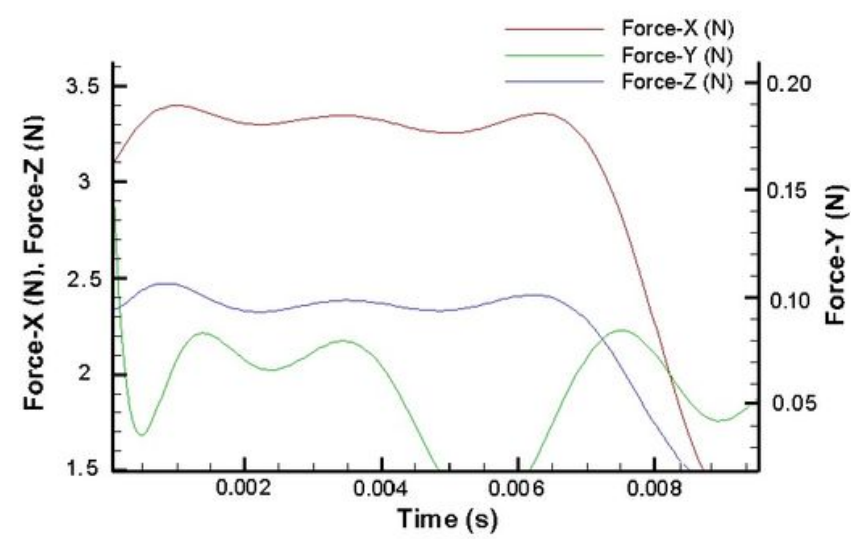

b. High-pressure water cooling.

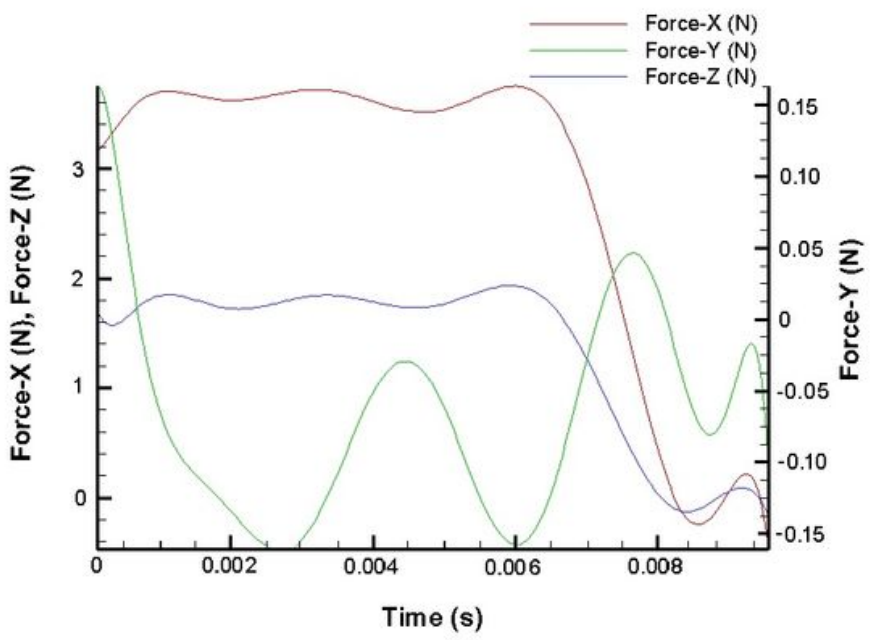

d. Low-temperature steam cooling.

Figure 10

Cutting force curve diagram under cooling medium.
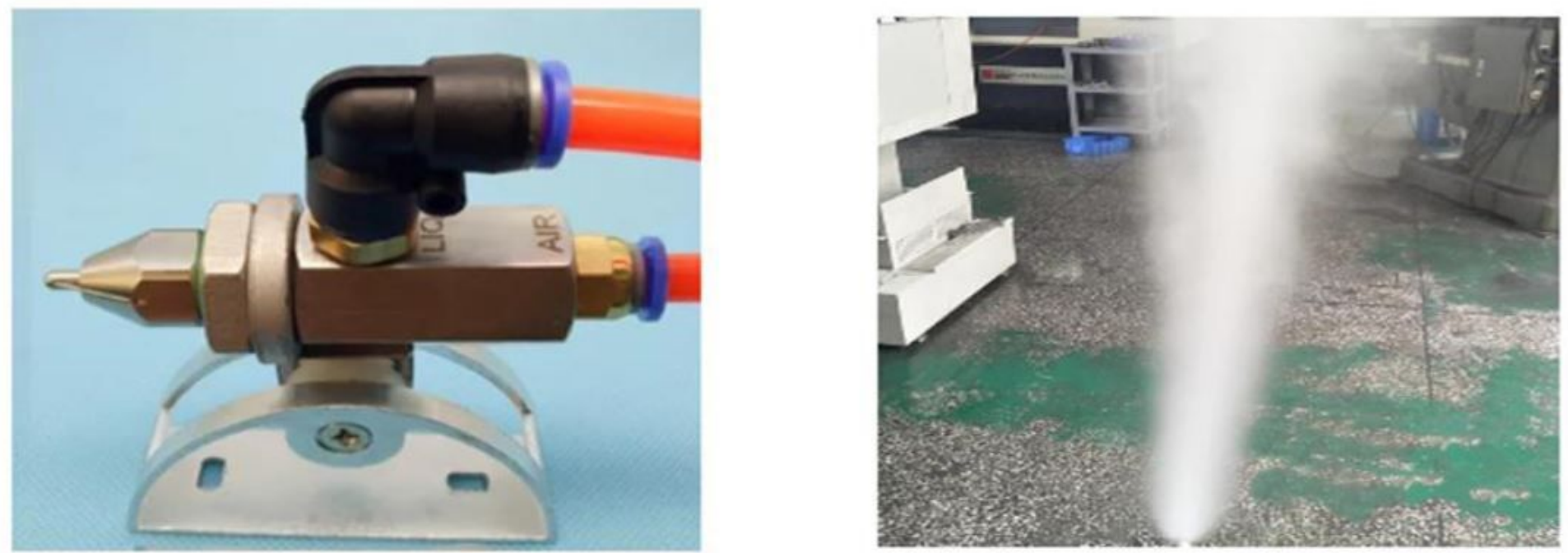
Low-temperature water atomization nozzle and atomization effect.

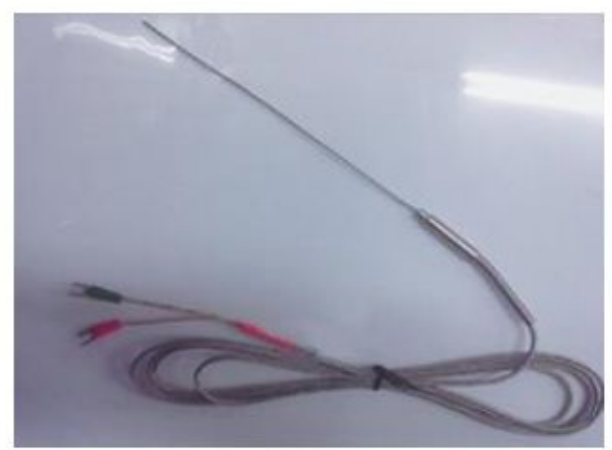

a. Thermocouple temperature sensor.

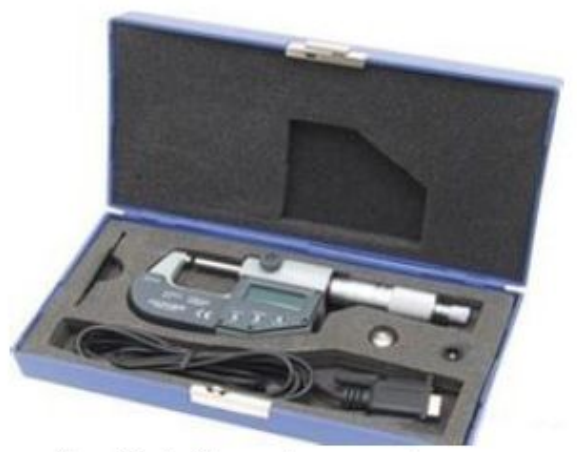

b. Outside micrometer.

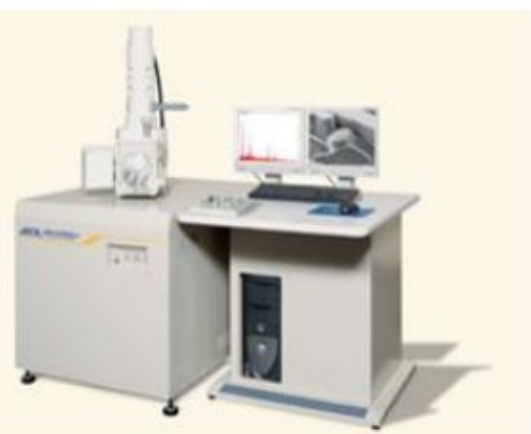

c. scanning electron microscope.

Figure 12

Experimental measuring equipment.

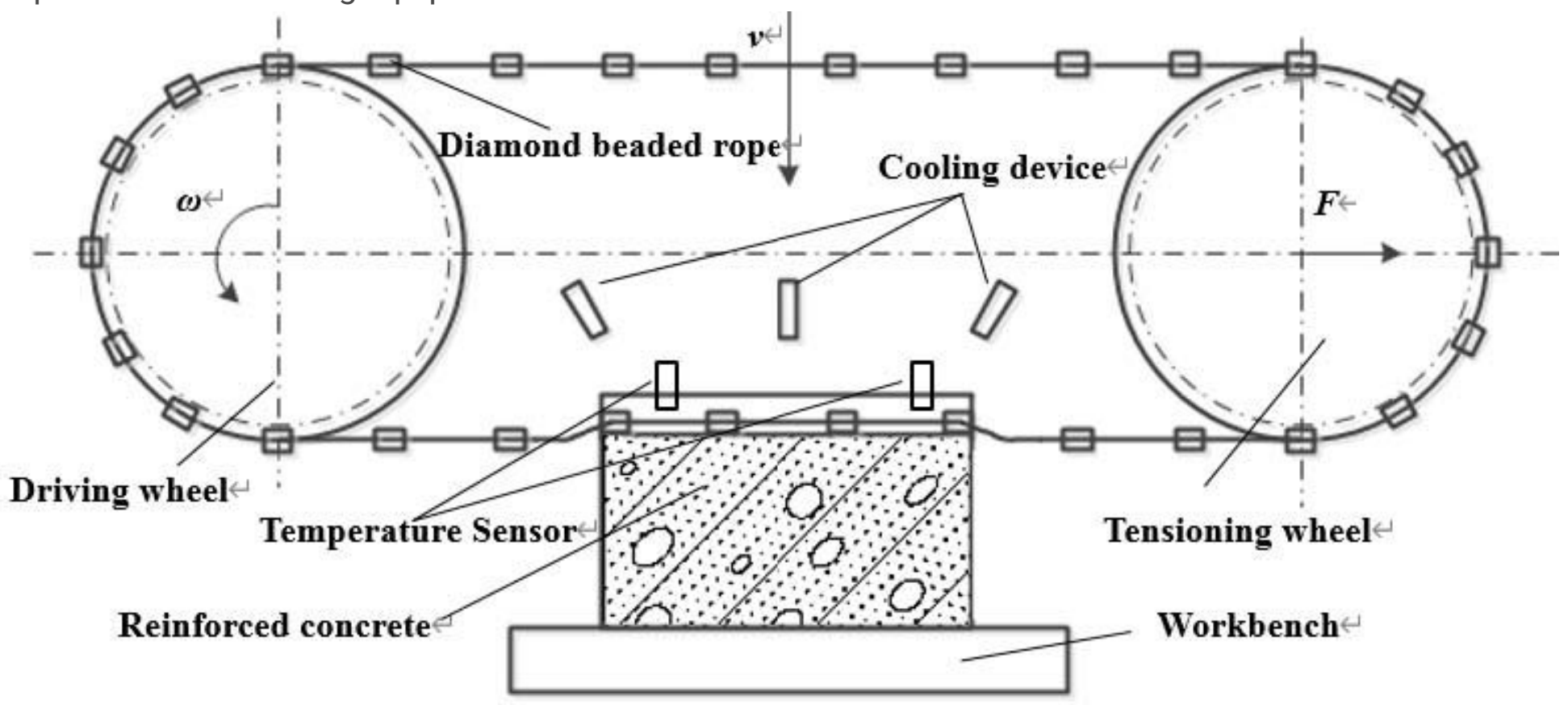

Figure 13

Schematic diagram of the experimental platform. 


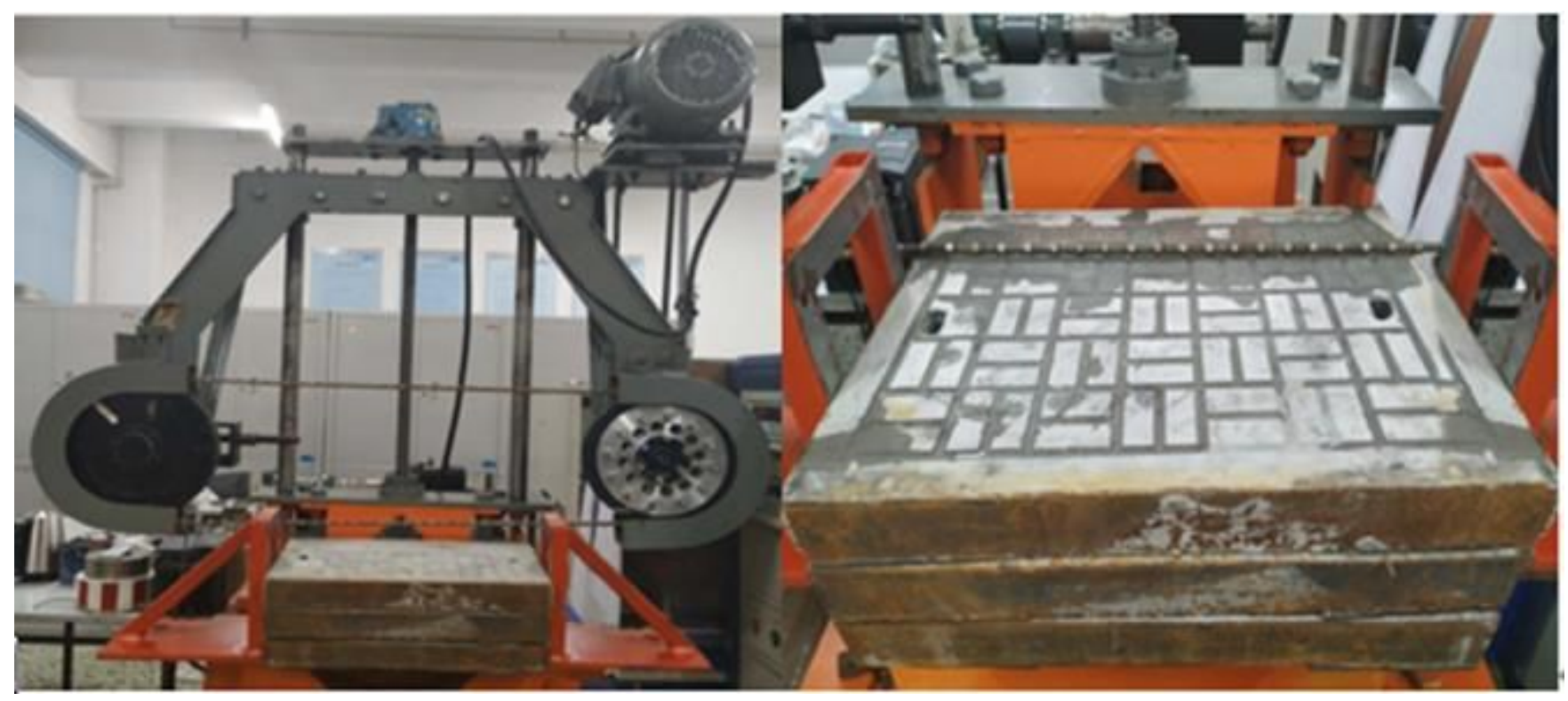

Figure 14

Experiment platform.

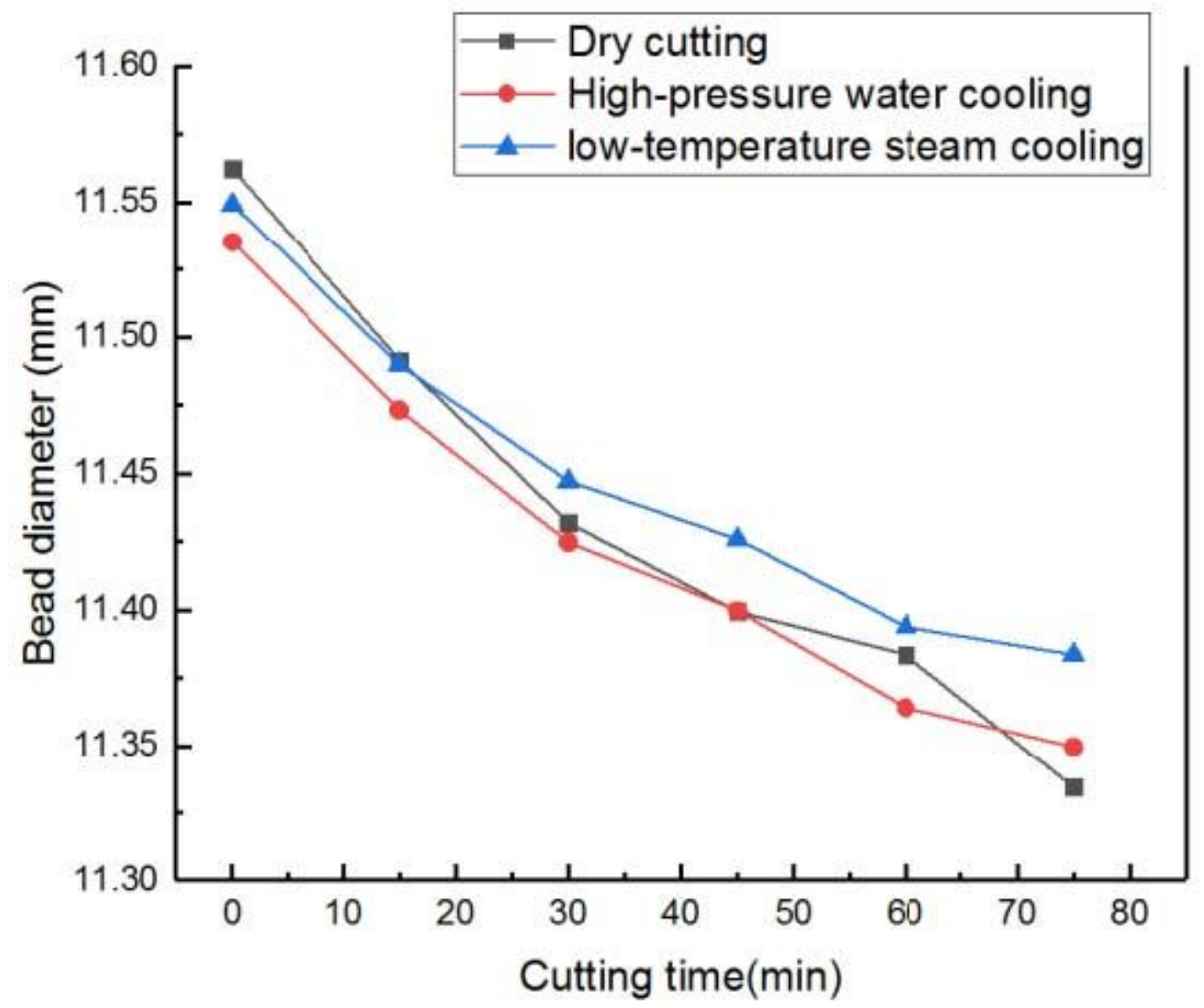

Figure 15

Variation curve of bead diameter under different cooling media. 


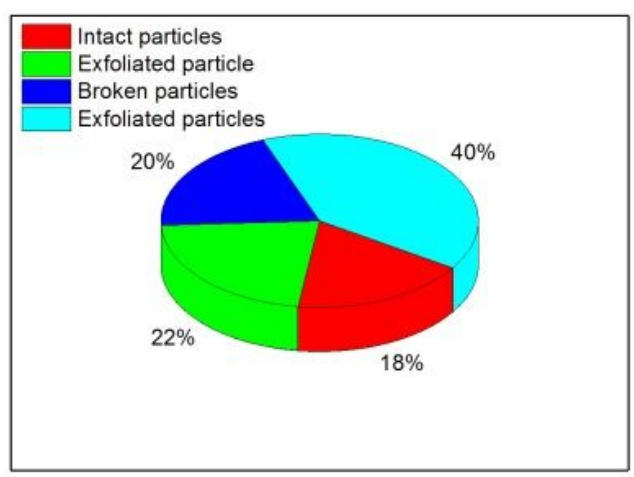

a. Dry cutting.

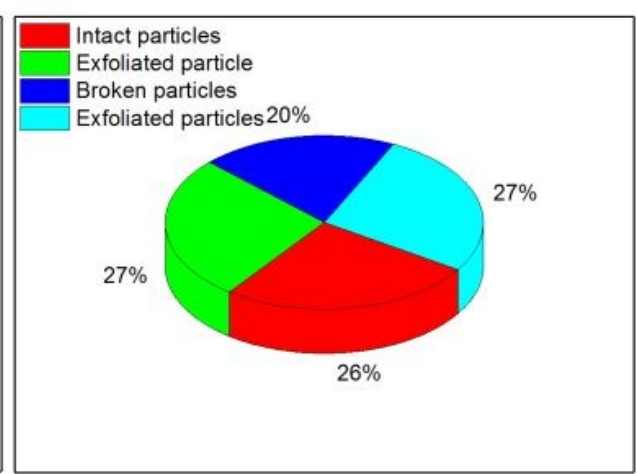

b. High-pressure water cooling.

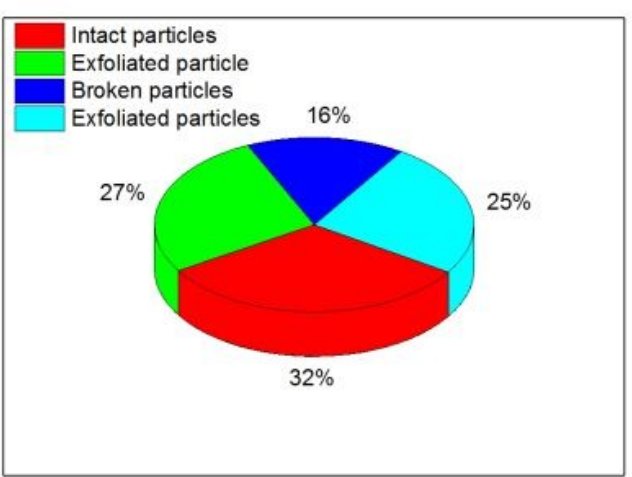

c. Low-temperature steam cooling.

\section{Figure 16}

The distribution ratio of diamond particles wear state under different cooling media. 